\title{
Evolution of Microgrids with Converter-Interfaced Generations: Challenges and Opportunities
}

\author{
Md Alamgir Hossain*1,2, Hemanshu Roy Pota ${ }^{1}$, Md Jahangir Hossain ${ }^{3}$, and Frede Blaabjerg ${ }^{4}$ \\ ${ }^{1}$ School of Engineering \& Information Technology, The University of New South Wales, Canberra, ACT-2610, Australia \\ ${ }^{2}$ Department of Electrical \& Electronic Engineering, Dhaka University of Engineering and Technology, Gazipur, Bangladesh \\ ${ }^{3}$ Department of Engineering, Macquarie University, Sydney, NSW-2109, Australia \\ ${ }^{4}$ Department of Energy Technology, Aalborg University, Denmark
}

\begin{abstract}
Although microgrids facilitate the increased penetration of distributed generations (DGs) and improve the security of power supplies, they have some issues that need to be better understood and addressed before realising the full potential of microgrids. This paper presents a comprehensive list of challenges and opportunities supported by a literature review on the evolution of converter-based microgrids. The discussion in this paper presented with a view to establishing microgrids as distinct from the existing distribution systems. This is accomplished by, firstly, describing the challenges and benefits of using DG units in a distribution network and then those of microgrid ones. Also, the definitions, classifications and characteristics of microgrids are summarised to provide a sound basis for novice researchers to undertake ongoing research on microgrids.
\end{abstract}

Keywords: Converter-based microgrids, distribution networks, renewable energy sources, definitions of microgrids and distributed generation units.

\section{Abbreviations}

The following abbreviations are used in this manuscript:

DGs Distributed generations

RESs Renewable energy sources

PECs Power electronic converters

DFIG Double-fed induction generator

PV Photovoltaic

PCC Point of common-coupling

5 MGCC Microgrid central controller

$\mathrm{CO}_{2} \quad$ Carbon dioxide

CHP Combined heat and power

HVDC High-voltage direct current

CSCs Current-source converters

VSCs Voltage-source converters

UPS Uninterruptible power supply

\footnotetext{
${ }^{*}$ Corresponding author

Email address: Md.Hossain6@student.adfa. edu .au or alamgir_duet@hotmail.com (Md Alamgir Hossain*1,2)
} 
EVs $\quad$ Electric vehicles

FACTS Flexible alternating current transmission systems

DSTATCOM Distribution static synchronous compensator

UPQC Unified power quality conditioner

THD Total harmonic distortion

MSAT Multiphase staggering auto-configured transformer

SVCs Static var compensators

CPDs Custom power devices

NPC Neutral point clamped

STATCOM Static synchronous compensator

APLC Active power line conditioner

PSO Particle swarm optimisation

MFD Morphological fault detector

HIL Hardware-in-loop

STS Static transfer switch

CERTS Consortium for electric reliability technology solutions

NEDO New energy and industrial technology development organisation

INESC TEC Institute for systems and computer engineering, technology and science

EU European union

LV Low-voltage

MV Medium-voltage

HV High-voltage

EMS Energy management system

IDE4L Ideal grid for all

PHEVs $\quad$ Plug-in hybrid EVs

MGCC Microgrid central controller

SCADA Supervisory control and data acquisition

MAS Multi-agent system

KnEA Knee-point-driven evolutionary algorithm

FCL Fault current limiter

SRCU Supervisory remote control unit

NDZ Non-detection zones

ICT Information and communication technology

PLCC Power line carrier communication

NN Neural network

DNO Distribution network operators

GA Genetic algorithm

IWD Intelligent water drops

ESSs Energy storage systems 


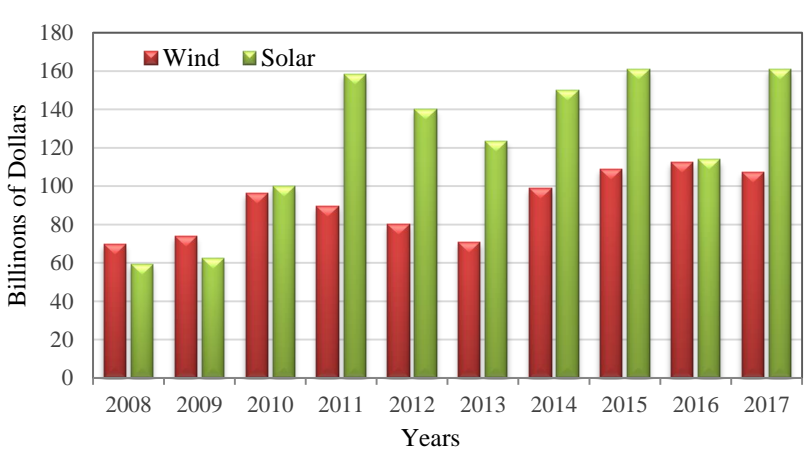

(a)

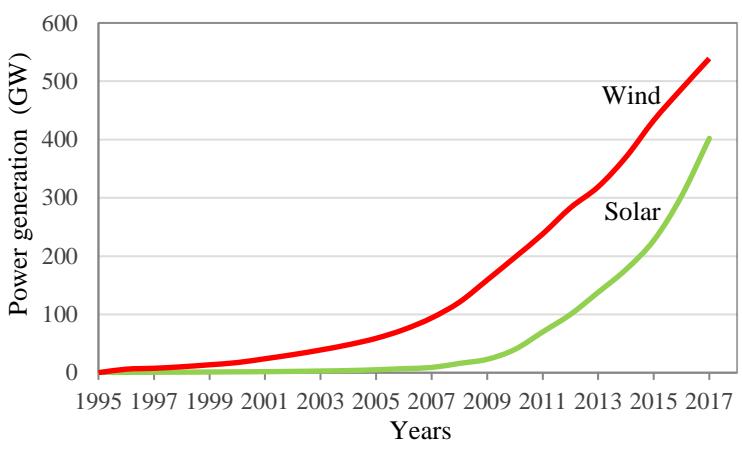

(b)

Figure 1: Wind and solar power: (a) global investment and (b) worldwide power generation.

$\begin{array}{ll}\text { ARMA } & \text { Autoregressive moving average models } \\ \text { NWP } & \text { Numerical weather prediction } \\ \text { MLIP } & \text { Mixed linear integer problem } \\ \text { ADP } & \text { Adaptive dynamic programming } \\ \text { I-DEMS } & \text { Intelligent dynamic energy management system } \\ \text { EDRP } & \text { Emergency demand response program } \\ \text { TOU } & \text { Time of use } \\ \text { RTP } & \text { Real-time pricing } \\ \text { CPP } & \text { Critical-peak pricing }\end{array}$

\section{Introduction}

Currently, there is a shift in power generation from conventional energy sources to renewable ones for a 
There is a number of literature review on microgrids from different perspectives, including components, controls, assessments and policies. In [5], different multilevel converters with newer topologies for the application of RESs, namely wind and solar, are presented. Some recently developed control strategies (inner-loop and primary) for operating them in an effective and efficient way, together with their relative advantages and disadvantages, are documented in [4. Adaptive and intelligent control methods for achieving microgrid stability and reliability are discussed in [6]. The stability of a microgrid based on the features of its components is assessed in [7]. In [8], a multi-agent system (MAS) for microgrid control and optimisation is reported. The basic structures of multi-microgrids with key technologies from the perspectives of voltage-grade classification as well as phase-sequence and ac/dc constitutional forms are discussed in [9]. In [10, microgrids are classified in terms of a hierarchical organisational scheme. The influence of government policy on the implementation of microgrids from the economic perspectives is presented in [11. In [12, the parameters of typical ac and dc microgrids are described, and some of the issues involved in their implementation and development are presented for the selection of a suitable configuration of a microgrid.

While there are many research or review papers on the control, operation and management of microgrids [5, 6. 8, 7, 10, 9, 11, 13, 4, 14, there is a gap in terms of identifying the definition and characteristics of converterbased microgrids and all their possible related challenges and opportunities to distinguish them from distribution networks. To bridge this gap, this paper offers the following contributions. As well as an updated review of the different issues concerning microgrids, the definitions and characteristics of state-of-the-art converter-based microgrids revised according to their specific roles are presented. The most distinguishing feature of this paper is a comprehensive analysis that differentiates a microgrid from a distribution network considering all possible issues to avoid the confusion that often arises regarding microgrid terms. Also, the very early developments of power systems and then the most advanced ones are discussed, with future possible research trends highlighted to assist novice researchers.

The rest of this paper is organised as follows. Section 2 briefly describes the development from traditional to modern power systems. In Section 3 , the advantages and disadvantages of using DG units in a distribution network are discussed. Section 4 presents the evolution, classifications, characteristics and definitions of microgrids, and also discusses their future challenges and opportunities. In Section 5 future trends in microgrids for upcoming researchers to advance the field is stated. Section 6 draws the conclusion.

\section{Background of modern power system}

In the early stages of electricity generation, generators were placed near the premises that consumed the power produced. With the increasing demand for electricity, the disadvantages of this approach became apparent, and it led to the evolution of centralised power systems. A centralised power generation station were installed to minimise costs and improve the security of supply [15. Prior to the 1990s, attempts to build increasingly larger power stations for financial benefits involved these stations being located either near fuel sources or in remote places for safety reasons. This indicates they were further away from the point of power consumption both electrically and geographically. Generally, in a large power station, a fossil fuel (coal, gas or oil) is burnt in a furnace to produce high-pressure steam which strikes a turbine's blades and rotates them, eventually causing a large synchronous generator to produce electricity in bulk (100 MW to $1 \mathrm{GW}$ ). Then, the 


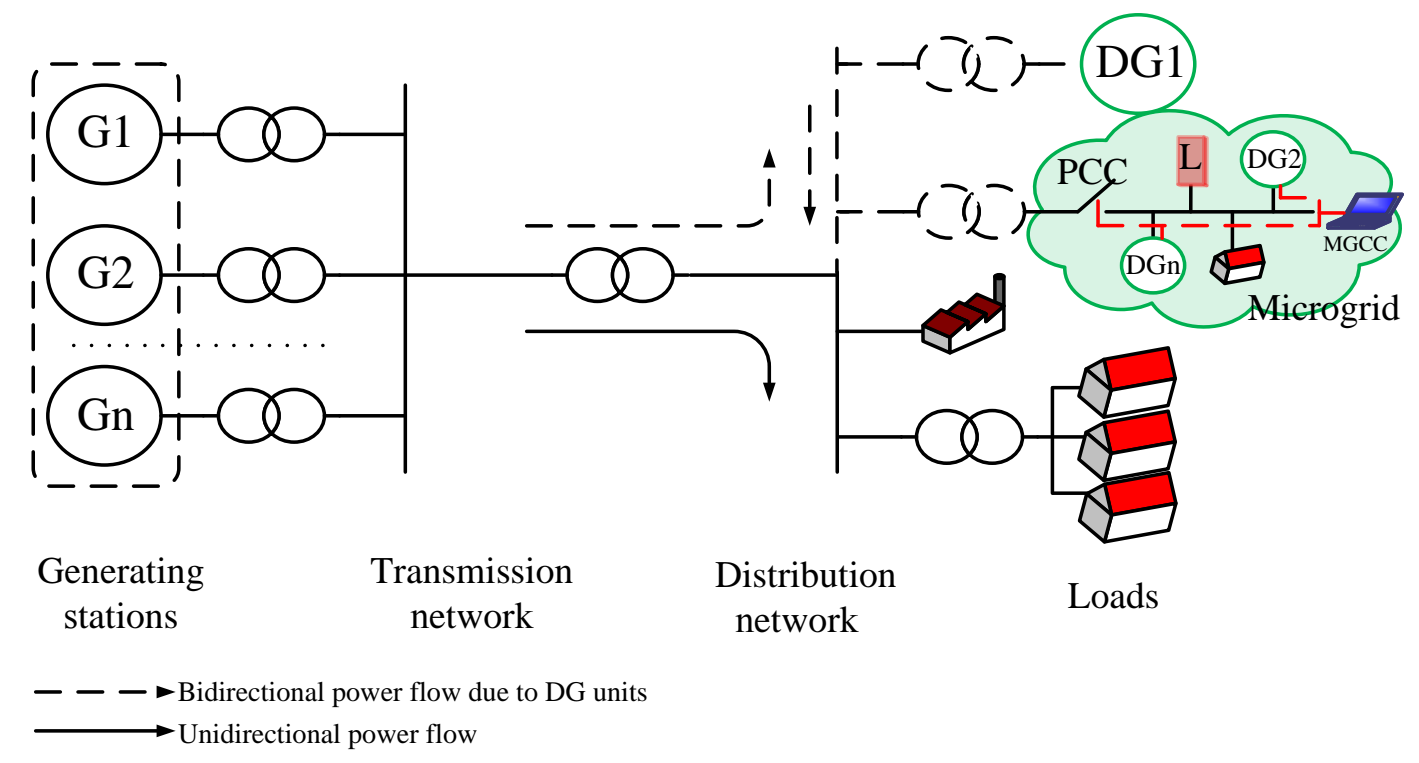

Figure 2: Evolution from traditional to modern power systems.

voltage of the generated power is stepped up by a transformer to a high-voltage (HV) to send the electricity long distances through transmission networks to substations where the voltage is stepped down to a medium and/or low-voltage (MV and LV, respectively) to reach end-users through distribution networks [16], as shown in Figure 2, Therefore, the generated electricity flows through transmission lines from large generation points to distribution points, i.e., it is a unidirectional (radial) power flow.

Since the 1990s, the construction of larger power systems has been discouraged for several reasons, including their increasing $\mathrm{CO}_{2}$ emissions, being energy deficient, lacking security of supply and incurring transportation losses [17, 18. Now, most governments provide financial incentives for the exploitation of renewable energybased power generation, the generators for which are, by nature, smaller than conventional ones. Therefore, small-scale power generators driven by RESs are increasingly being directly connected to distribution networks whereby consumers have direct access to electricity generation. These small-scale units are defined as distributed generation (DG) units.

Incorporating DG units in a distribution network represents a fundamental change from a traditional power system as the resultant power flow is bidirectional as shown in Figure 2, Although these units are based mainly on RESs, such as solar and wind generators, they can rely on other sources, such as micro-turbines, gas turbines, fuel cells, biogas and combined heat and power (CHP) units [19, 20, 21]. DG units driven by RESs have demonstrated significantly increased electricity generation over the last two decades [22], as shown in Figure $1 b$, The integration of these sources in a distribution network demonstrates both merits and demerits for the existing power system.

\section{Benefits and challenges of using DG units in distribution networks}

\subsection{Benefits of using DG units}

DG units, which are small generators, offer numerous advantages compared with conventional large power plants. Some of their key features described in the following subsections emphasise the importance of current research [23, 24, 25, 26, 27, 28]. 
Reliable electricity supply: As explained in Section 2 in a traditional power system, a generator produces bulk power (500 MW) in one place and transmits it to other places (end-users, i.e., consumers) to service a large community. On the other hand, a DG unit, the rating of which is typically low (less than 5 MW), produces electricity and services for a local small community. In the case of the failure of a 500 MW generator, the system needs to either produce an extra $500 \mathrm{MW}$ of power from reserve units or shed a $500 \mathrm{MW}$ load to save the system from a cascading generator shutdown. In contrast, power failures in DG units have less impact on the system and are often manageable because of the small-scale power generation [24].

Reduced transmission losses: Electrical power flows from a power station to consumer premises through transmission lines which have inherent resistance and reactance that cause them to lose power. If $142 \mathrm{~A}$ current flows through a line as shown in Table 1, a $0.642 \Omega \mathrm{km}^{-1}$ resistance in a line leads to a $12.95 \mathrm{~kW} \mathrm{~km}^{-1}$ loss. Installing DG units near consumer premises can minimise $\mathrm{I}^{2} \mathrm{R}$ losses which improves network efficiency [25].

Reduction in feeder capacity: The gradually increasing demand for power as a result of the desire to maintain high living standards for an increasing population necessitates changing the components of a power system used in transmission lines. The penetration of DG units in a distribution network reduces stresses on both transmission line equipment and distribution lines at times of peak demand [26]; for example, as patterns of daily office loads are more or less similar to the generation of solar power, the need to import power from the grid is avoided which places less stress on transmission lines. Therefore, using DG units, which encourage more load participation in a distribution network, can be more economical than upgrading a network's components.

Integration of RESs: The majority of DG technologies used in a network are based on RESs which have abundant input power and do not emit $\mathrm{CO}_{2}$, i.e., they are environmentally friendly. Installing DG units around load centres provides an enhanced voltage profile and better power quality [27]. Research on DG technology is gradually reducing their production costs and increasing the power conversion efficiency of DG units, thereby leading to their being attractive choices for future power generation.

\subsection{Challenges of implementing DG units}

Conventional electricity structures have accommodated the installation of DG technologies in a distribution network although this can affect various quantities of a power system, such as its voltage profile and power flow. Important concerns regarding power quality, changes in a network's voltage and fault levels, system stability, modelling of networks' components and low inertia for the operation of DG units in a distribution network are briefly discussed in the following subsections.

Power quality: The power quality of a distribution network can be altered by the penetration of DG units which, if operated based on RESs, may lead to problems such as voltage fluctuations, harmonic distortions, frequency deviations and voltage flickers 29]. This is because the variable outputs of DG units cause reverse power flows, and the use of many PECs produces harmonics in the injected power. Also, the connection and disconnection of a DG unit to/from a network may cause transient currents which lead to a high-magnitude voltage [30]. Voltage flicker can be minimised by imposing constraints on how and when DG units change their outputs while a great deal of attention has been paid to increasing the power quality of a network during voltage disturbances [31, 32. The power quality of a distribution network can be improved in several ways, including by applying better control strategies for the inner-current control of converters, designing filters for interfacing converters, applying compensators and installing flexible alternating current transmission systems 

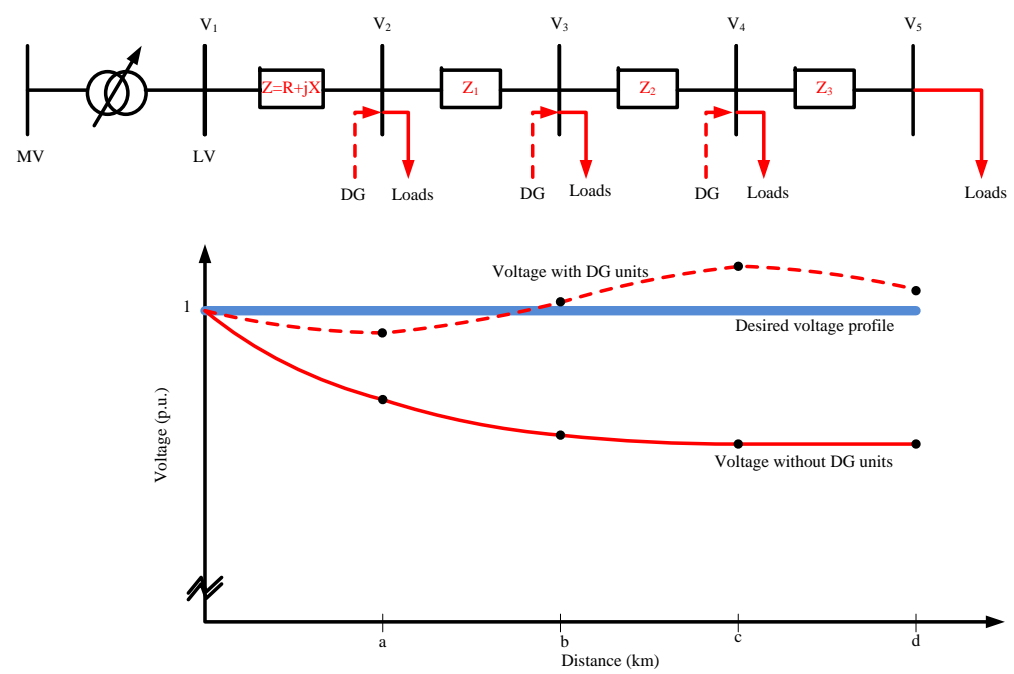

Figure 3: Impact of DG integration on voltage profile of a radial distribution network.

(FACTS) devices in networks [33, 34].

Power quality is generally enhanced by the installation of devices at customers' supply points, such as a series compensator (dynamic voltage restorer (DVR)), a shunt compensator (distribution static synchronous compensator (DSTATCOM)), and hybrid series and shunt compensators (unified power quality conditioner (UPQC)). A series compensator is employed for voltage-related issues and a shunt compensator for minimising current-related ones, such as the effects of an imbalance, harmonics and the power factor. A dual-tree complex wavelet transform-based control algorithm for a DSTATCOM to enhance power quality in a distribution network is presented in 34. The total harmonic distortion (THD) in this method is less than $5 \%$ with the application of various load conditions under the unity power factor. A 20-pulse multiphase staggering autoconfigured transformer (MSAT), a combination of delta and zig-zag transformers, to improve power quality in MV and high power applications is discussed in [35].

Change in network voltage: The main function of a DG unit is to supply power within acceptable voltage ranges to customers. Although the standards for voltage profiles vary from country to country, their operating principles are the same as that of radial feeders which are generally arranged in such a way that the power flow is unidirectional, i.e., the power flows from the generator to the load. As a result, a poor voltage profile as shown in Figure 3 is observed at the most remote node because of the voltage drop in impedance along the radial lines. This problem can be minimised by inserting DG units directly into a network; however, this causes a bidirectional power flow along the lines that require complex protection schemes for the network 36. If the impact of these variations in a radial network is significant, the locations, sizes and impedances of these units must be appropriately measured 36 .

The insertion of DG units can also vary the voltage profile due to the intermittency of the power generated by RESs, especially on cloudy days or at times of low wind velocity, with a voltage collapse possibly leading to a complete interruption of power 37. To inject or absorb reactive power in a network, shunt capacitor banks and static var compensators (SVCs) or FACTS devices need to be placed at optimal positions in the network 38 . Due to smaller size and faster response, a static synchronous compensator (STATCOM) is preferable to a SVC. In 39, a survey of the literature is presented on the optimal allocation of a D-STATCOM in a distribution 
network. The real-time control of multiple custom power devices (CPDs) using STATCOM and active power line conditioner (APLC) to improve voltage regulation in a distribution network are demonstrated in [37. In this strategy, two particle swarm optimisation (PSO) algorithms are implemented to obtain the optimal sizing/siting and online control of multiple CPDs. In [40, the operational performance of a low-capacitance cascaded H-bridge STATCOM is optimised to minimise switching losses.

Change in network fault level: A distribution network containing DG units is an active network in which the power and extra current can flow in a reverse direction toward the fault side [41, with all types of DG units assisting in increasing the fault level. Although synchronous and induction generators are capable of supplying a fault current, inserting DG units can alter the fault level. Then, if all the associated protection apparatuses are upgraded to maintain the equivalent fault level, costs will increase. Although involvement of the fault level can be restricted by using an appropriate impedance, this technique augments power losses and voltage changes 42 .

A robust and versatile algorithm for over-current protection considering changes in power generation and demand is described in [43. Faults are detected by studying abrupt changes in the current's magnitude using a multi-stage morphological fault detector (MFD). In [44, a hardware-in-loop (HIL) adaptive protection scheme capable of addressing the DG effect by providing a flexible protection solution is introduced. As an adaptive protection scheme depends on communication lines to adjust the relay setting, a communication failure becomes a major threat to its security. Such a scheme that takes into account a super-capacitor's energy to improve resilience against communication failure is presented in [45]. In [46, a current limiting-approach and generalised fault model for enhancing the fault ride-through capability of droop-controlled converter-interfaced DG units are presented. A dc-link adjustable resistive type of fault current limiter for improving the fault ride-through capability of a voltage-source converter (VSC) is presented in [47, 48.

System stability: An interconnected power system, which is generally large, creates complex networks in which different factors, including uncertainties in power generation and demand, can lead to instability. As power generations from RESs highly reliant on weather conditions, several transient disturbances, which are more complex and distributed than the existing ones, including a loss of power generation or system components as well as faults, increase in a distribution network.

The stability of a network is generally concerned with its transient stability, ability of a system to return back to its steady state when subjected to a large disturbance. In 49, the transient stability in Dutch 10 $\mathrm{kV}$ distribution network with micro-turbines, CHP plants, and wind generators is analysed. It is concluded that DG units have transient stability issues in a network which should be taken into account during their integration. A projective integration method considering a high penetration of DG units for the improvement of the transient stability of a distribution network is described in [50. In [51, the effect of a network's transient stability on its reliability during dynamic contingencies is assessed using a direct method. A robust transient stability-constrained optimal power-flow model that takes into account uncertain variations in the model's parameters and dynamic loads is presented in [52. A fuzzy-based damping controller that uses the frequency to control the firing angle of a thyristor-controlled series capacitor to enhance the damping of electromechanical oscillations in a distribution network is presented in 53 . The effects of a power system stabiliser and oscillation modes of a distribution network are studied in [54.

Modelling of network's components: Generally, the dynamic behaviours of a power system are analysed 
Table 1: Typical line parameters.

\begin{tabular}{|l|c|c|c|c|}
\hline Type & $\mathrm{R}[\Omega / k m]$ & $\mathrm{X}[\Omega / k m]$ & $I_{N}(\mathrm{~A})$ & $\mathrm{R} / \mathrm{X}$ \\
\hline $\mathrm{LV}$ & 0.642 & 0.083 & 142 & 7.73 \\
$\mathrm{MV}$ & 0.161 & 0.190 & 396 & 0.85 \\
$\mathrm{HV}$ & 0.060 & 0.191 & 580 & 0.31 \\
\hline
\end{tabular}

Table 2: Comparison of different models 65.

\begin{tabular}{|l|l|l|l|l|}
\hline Model & Operating point & Small-signal & High frequency & Non-symmetric network \\
\hline Time-varying phasors & Yes & Yes & No & No \\
Dynamic phasors & Yes & Yes & - & - \\
$a b c$ & No & No & Yes & Yes \\
$d q 0$ & Yes & Yes & Yes & No \\
\hline
\end{tabular}

by using time-varying phasor models (quasi-static models), often by means of network power flow equations, that assume the frequency of signals is approximately constant [55. Consequently, models are time-invariant, with a well-defined operating point that facilitates the power system study. However, due to increasing penetration of RESs into an existing distribution network, it becomes complex to observe the dynamic behaviours. This is because of the introduction of power electronics, involved with harmonics in voltage and current signals that may exhibit fast amplitude and phase variations [56]. As a result, the system may not be quasi-static in many cases. To facilitate the analysis, several alternative models, including dynamic phasors, $a b c$ and $d q 0$ reference frame models, are developed [57. In dynamic phasors, voltage and current signals are represented by Fourier series expansions that evaluate harmonic components over a moving time window [58, 57]. It approximates the system with nearly periodic quantities [59]. Different dynamic phasor based models of a power system are demonstrated in [57]. The time-based model using the $a b c$ reference frame is often used in power systems for application of unbalanced networks, where frequency is not constant. In $d q 0$-based models, the symmetric electrical quantities are mapped into constant values. The assumption of quasi-static states is not required in this model, and it can work at high frequencies [60]. A number of studies are presented based on $d q 0$-based models for analysing the network's dynamics 61, 62, 63, 64. A comparison among different models is shown in Table 2 ,

Low inertia: In a conventional generator, the inertia as an energy - a form of kinetic energy stored in a rotating mass of a synchronous generator - can support regulation of the network's frequency and voltage up to 10 seconds during disturbances that lead to a generation-demand imbalance 66. This inertia energy basically minimises the impact induced from disturbances within the time frame, whereas other controllers take initiatives after this step. However, the increasing penetration of converter-fed RESs that have non-existent or lower inertia characteristics than synchronous generators reduces the inertia of an entire network, leading to voltage or frequency fluctuations during an event [67, 68. If appropriate control measures are not applied to minimise these fluctuations within specified limits, the network may experience instability [69]. Several control strategies that realise the role of inertia in a network have been developed from different perspectives; for example, in a solar generator, the operating point of a PV voltage can be increased beyond the maximum power-point voltage 
to enable the reserve power to act as an inertia energy during frequency or voltage fluctuations [70]. In the case of a variable-speed wind generator, the rotor's speed and blades' pitch-angle controls can be used to reserve the inertia power to respond to any change in the network [1]. Also, the kinetic energy of rotating blades can be released using an appropriate control strategy for converters to respond in frequency deviations up to $2-6 \mathrm{~s}$ 72. Although these techniques, called de-loadings, can reserve some energy at the expense of constant energy losses to emulate inertia behaviour, they may still cause reliability issues due to their reliance on the variable factors of RESs.

To improve the reliability of a network, its low inertia can be enhanced by using batteries, capacitors and flywheels at the dc busbars of its interfacing converters. A battery can be regarded as a reserve energy source that responds to a network's requirements, i.e., it can store or release energy according to the power demand 73. In [74, an ESS used as an inertia energy to regulate the frequency of a microgrid has the capability to seamlessly transfer. A capacitor employed to decouple a converter's input power from the boost converter's output can be used as an inertia of the converter to control the terminal voltage [1. In order to apply the well-established conventional control algorithms/theories to converter-interfaced generators, the concept of a synchronverter, which mimics the behaviours of conventional synchronous ones over short periods of time for the integration of RESs in a distribution network, is proposed in [75]. The amount of inertia depends on the size of the virtual moment of the synchronverter's inertia with capacitors typically used to mimic it. However, to obtain a better performance that resists the intermittency of RESs, relatively large storage is preferable. An improved synchronverter that limits its voltage and frequency to within acceptable ranges is presented in [76. In [77, algorithms for a synchronverter are modified to include the virtual field current, virtual filter inductors, (virtual) nominal active mechanical torque and virtual capacitors to enhance its performance and stability, and bring the inverter's characteristics much closer to those of synchronous machines. The hybrid use of a battery and ultracapacitor, where the latter handles high-frequency and the former low-frequency power fluctuations to implement a virtual synchronous generator, presented in [78] reduces power fluctuations. Researchers have still opportunities to improve the inertia response by the application of novel control strategies and algorithms, and the design of hybrid energy storage systems(ESSs).

\section{Microgrids}

\subsection{Evolution of microgrid concept}

To exploit the benefits of integrating DG units and overcome the issues related to the management of a network's components - DG units, loads and other equipment - from a grid utility perspective because of their multi-dimensional activities, the concept of a microgrid has evolved [79]. The word 'microgrid' is comprised of 'micro' (an extremely small attribute from the perspective of a grid utility) and 'grid' (a network of electric lines). Therefore, it is expected that microgrids will possess all the essential components of a power system in a small range, except some unnecessary equipment, such as transmission lines and substations. The core idea of a microgrid is to integrate a limited number of DG units to optimally control DG units without creating a complex network. Its major components are a hierarchical control approach, a point of common coupling (PCC), distributed controls using local information and a specific region that enable DG units to be integrated in a systematic way to ensure reliable operation of the system. As it consists of DG units, loads, energy storage 
Table 3: Comparison of microgrids and modern distribution networks.

\begin{tabular}{|c|c|c|c|c|c|}
\hline Descriptions & Microgrids & Distribution networks & Descriptions & Microgrids & Distribution networks \\
\hline Central control unit & Yes & No & Power supply security & Yes & No \\
\hline Specific areas & Yes & No & Monitoring system & Yes & No \\
\hline Point of common coupling & Yes & No & Stability & High & Low \\
\hline Categorising loads & Yes & Yes & Storage system & Yes & Yes/No \\
\hline Communication with grid & Yes & Yes & Dynamic electricity prices & Yes & Yes/No \\
\hline High penetration of RESs & Yes & No & Self-healing system & Yes & No \\
\hline Adequate protection & Yes & Yes & Systematic structures & Yes & No \\
\hline Seamless transfer & Yes & No & Flexible operation and management & Yes & No \\
\hline Decentralised control system & Yes & Yes & Uninterruptible power supply & Yes & No \\
\hline Systematic energy exchange & Yes & No & Controllable and coordinated mode & Yes & No \\
\hline Communication facilities & Yes & Yes & Low environmental impact & Yes & Yes/No \\
\hline Power outage & Low & High & Grid modernisation enabled & Yes & No \\
\hline Improved power quality & High & Low & Integration enhanced & Yes & No \\
\hline Reliability & High & Low & End-user needs met & Yes & Yes \\
\hline Supplementary power & Yes & No & Plug-and-play & Yes & No \\
\hline Optimum power management & Yes & No & Local power management & Yes & No \\
\hline Resilience of power supply & Yes & No & & & \\
\hline
\end{tabular}

elements, protection equipment and a control scheme, all of which ensure the regulation of the power flow, and voltage and frequency profiles within a defined region, it could be called a 'distribution network control' approach. However, as this description does not cover any specific region, it is not appropriate. Therefore, the integration of DG units in a distribution network to export power cannot be considered a microgrid unless it has control over the network within a defined region. The microgrid concept and distribution network are depicted in Figures 4 and 5 , respectively, and their relative features presented in Table 3 . One important point is that encouraging the construction of microgrids is not contrary to using a conventional power system but, rather, supplementary to a grid utility.

To obtain good services from a grid utility and DG units, microgrids are connected to the utility through a static transfer switch (STS) at the PCC. This approach provides control of the participating equipment within a defined region and enables the microgrid to be considered a single dispatchable unit from the grid perspective. An individual owner of a microgrid can both supply power to a grid utility if the microgrid has excess power and receive power if necessary. In a microgrid, generators deliver power at a certain voltage level during parallel operation with the grid utility and provide sufficient power to, at least, critical loads. Ensuring a reliable power supply for certain loads is the most important priority of a microgrid.

A microgrid can be disconnected during a period of deteriorating power quality or network contingencies without affecting the local power supply. A degradation in power quality may occur during a violation of the voltage limit of sensitive loads because, if the voltage quality hampers the equipment's operation, only local DG units can supply power to these loads. When, as an entity, a microgrid faces an unbalanced power supply, it can intentionally island itself from the grid utility to retain the power quality required for sensitive loads. This capability is effective for providing an uninterruptible power supply (UPS) to sensitive loads, not only when there is a loss of the main power supply and/or a grid failure but also during a period of the power degradation. A microgrid can be installed in any industrial area, official building, commercial centre or residential complex to receive UPS services.

Over the last decade, microgrids have attracted the attention of industry leaders and researchers, with some 
Table 4: Some global projects involving converter-based microgrids [80].

\begin{tabular}{|c|c|c|c|c|}
\hline Name/Place & Organisation/Institution & DG & Type & Load \\
\hline Aichi/Japan & NEDO & PV, Fuel Cell and Battery & $\mathrm{AC}$ & Industrial and Commercial \\
\hline Albuquerque/US & NEDO & PV and Battery & $\mathrm{AC}$ & Residential/Commercial \\
\hline Benchmark Low-voltage/Greece & Microgrids Project & PV, Wind, Fuel Cell, Flywheel and Battery & $\mathrm{AC}$ & Residential \\
\hline Barcelona/Spain & IREC & PV, Wind, EV and Battery & $\mathrm{AC}$ & HVAC, Air Conditioners \\
\hline Borrego Springs/US & San Diego Gas \& Electric & PV, EV and Battery & $\mathrm{AC}$ & Residential and Industrial \\
\hline California/US & Santa Clara University & PV and Wind & $\mathrm{AC}$ & University \\
\hline Chicago/US & Illinois Institute of Technology & PV, Wind, EV and Battery & $\mathrm{AC}$ & - \\
\hline Chico Mendes/Brazil & Electrobas & PV and Battery & $\mathrm{AC}-\mathrm{DC}$ & - \\
\hline Continuon Holiday Park/Netherlands & More Microgrids Project & PV and Battery & $\mathrm{AC}$ & Residential \\
\hline FIU Testbed/US & Florida International University & PV, Fuel Cell, Wind and Flywheel & DC & Residential and Motor \\
\hline Fukuoka/Japan & Smart Energy Laboratory & Wind, PV and Battery & DC & - \\
\hline Gazi University/Turkey & Gazi University & PV, Wind and Battery & $\mathrm{AC}$ & University \\
\hline Hawaii Hydrogen Power Park/US & Hawaii National Energy Institute & PV, Wind and Fuel Cell & $\mathrm{DC}$ & Residential \\
\hline Ilha da Ferradura/Brazil & - & PV and Battery & $\mathrm{AC}$ & - \\
\hline Kyoto Eco Energy/Japan & NEDO & PV, Wind, Fuel Cell, Biogas and Battery & $\mathrm{AC}$ & Residential \\
\hline Laboratory scale MG Testbed/US & New Jersey Government & PV and Battery & $\mathrm{AC}$ & Residential and Motor \\
\hline Los Alamos/US & NEDO & PV and Battery & $\mathrm{AC}$ & Residential \\
\hline Lyon/France & NEDO & $\mathrm{PV}$ and $\mathrm{EV}$ & $\mathrm{AC}$ & - \\
\hline Manzanita Hybrid Power Plant/US & - & Wind, PV and Battery & $\mathrm{AC}$ & - \\
\hline Nanjing University/China & Nanjing University & PV, Wind and Battery & $\mathrm{AC}$ & Motor \\
\hline RIT Microgrid/US & Rochester Institute of Technology & PV, Fuel Cell and Wind & $\mathrm{AC}$ & Residential and Motor \\
\hline Sino-Danish Project/China & Aalborg University/Tsinghua University & PV, Wind and Battery & $\mathrm{AC}$ & - \\
\hline UTC/France & University of Technology of Compigne (UTC) & PV, Fuel Cell and Battery & $\mathrm{DC}$ & Motor \\
\hline NTUA/Greece & National Technical University of Athens (NTUA) & PV, Wind and Battery & $\mathrm{AC}$ & - \\
\hline University of Nottingham/England & University of Nottingham & Wind and Battery & $\mathrm{DC}$ & Residential \\
\hline University of Miami Testbed/US & University of Miami & PV, Fuel Cell and Battery & $\mathrm{DC}$ & University Residential \\
\hline Woodstock/US & National Renewable Energy Laboratory & PV, Wind and Battery & $\mathrm{AC}$ & Shop and Office \\
\hline Xcalak/Mexico & - & Wind and Battery & $\mathrm{DC}$ & Village \\
\hline
\end{tabular}

Table 5: Some research projects conducted on microgrids 81 .

\begin{tabular}{|l|l|l|l|}
\hline Project Title & Budget & Duration & Promoter \\
\hline CERTS & $12.2 \mathrm{M}$ USD & from 1999 & US \\
More Microgrids & $8 \mathrm{M} €$ & 2006 to 2010 & EU \\
IDE4L & $8 \mathrm{M} €$ & 2013 to 2016 & EU \\
Microgrids & $4.5 \mathrm{M} €$ & 2003 to 2006 & EU \\
Positas Microgrid & $1.5 \mathrm{M}$ USD & from 2015 & US \\
Microgrid - Alstom & $1.2 \mathrm{M}$ USD & from 2014 & US \\
\hline
\end{tabular}




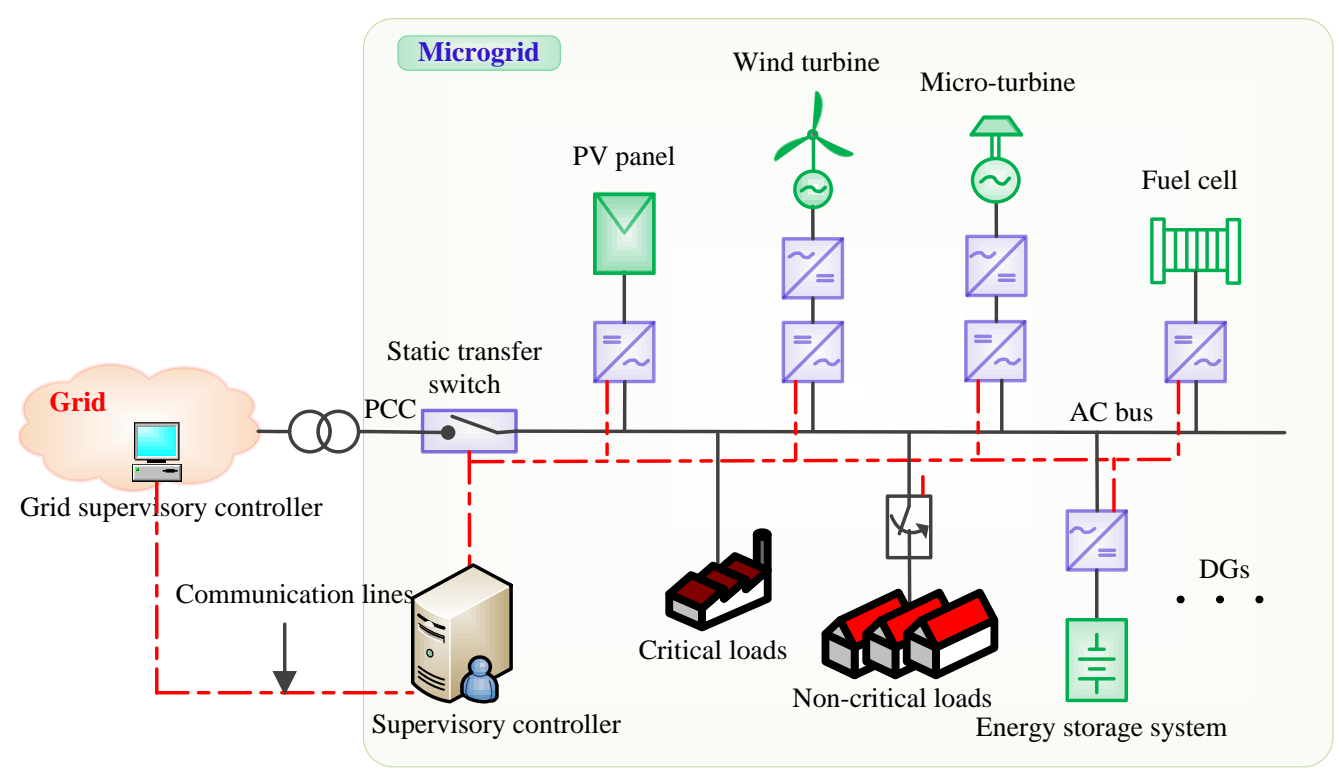

Figure 4: Concept of microgrid.

research projects shown in Tables 4 and 5 . Many of these were developed for test beds around the world; for example, the Consortium for Electric Reliability Technology Solutions (CERTS) in the United States of America (USA); the New Energy and Industrial Technology Development Organization (NEDO) in Japan; and The Institute for Systems and Computer Engineering, Technology and Science (INESC TEC) in Europe 82, 80, 83]. Some were implemented by governments to provide secure, clean and efficient energy; for example, in the USA, the Department of Energy spent 6.3 billion USD in the fiscal year 2012 on research, deployment and development activities related to clean energy 81 .

\subsection{Characteristics of Microgrids}

A microgrid has the following characteristics which distinguish it from DG units applied in a distribution network [79, 84, 85, 86, 87, 88, 89, 90.

- It supervises the electrical components, such as powers, voltages and frequencies, by means of monitors.

- It has a PCC in a distribution network for connecting and disconnecting the grid utility.

- It is a subset of LV or MV distribution networks.

- It consists of generation units, a hierarchical control approach, power consumption places and ESSs.

- It facilitates an UPS to, at least, the highest priority loads during a grid failure or power quality degradation.

- It has two operating modes: 1) grid-connected; and 2) islanded or standalone (autonomous).

- It acts as a single controllable entity from a grid perspective.

- It generates the required reference voltage and frequency in an islanded operation.

- It is constructed in a defined location which can be in a distribution network or remote area. 


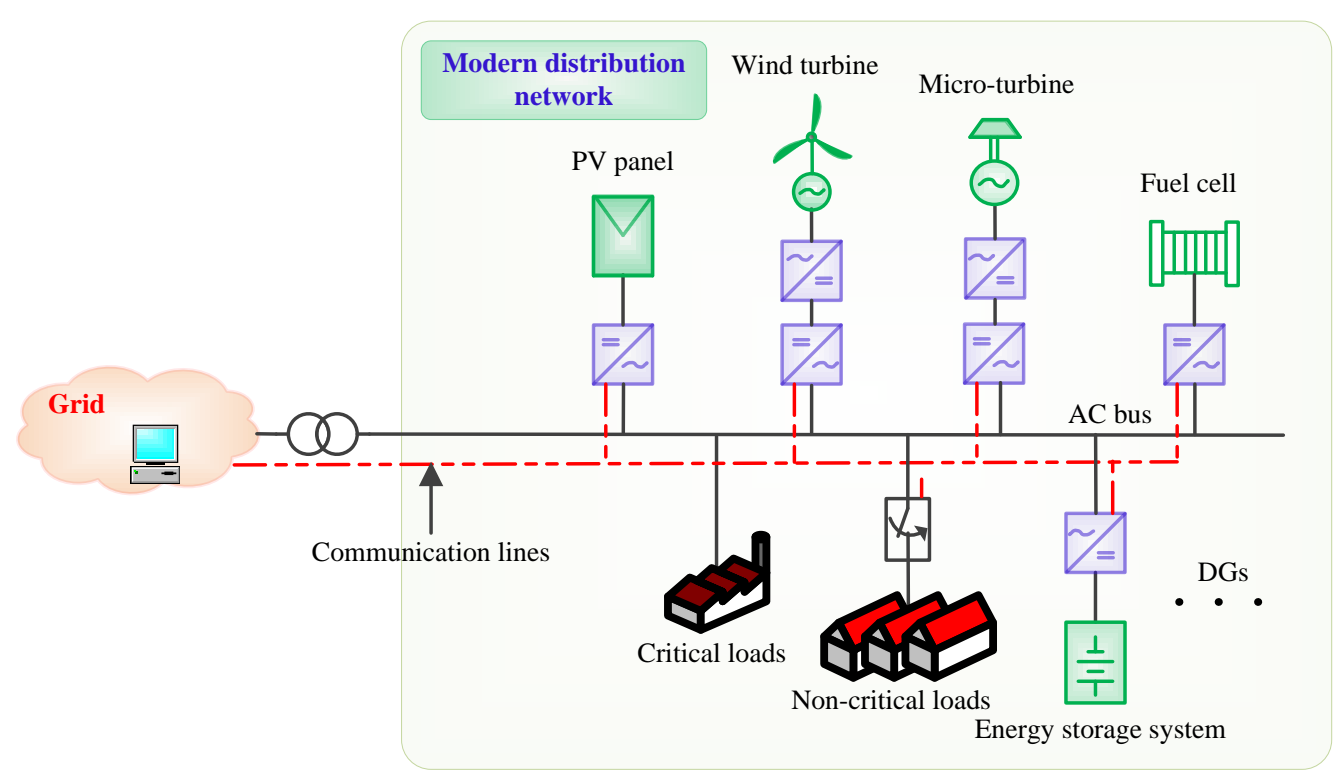

Figure 5: Modern distribution network.

- It has the necessary protection schemes.

- It controls the power supply during both grid-connected and islanded operations.

- It accumulates DG units, the ratings of which are less than $100 \mathrm{MW}$.

- It displays 'plug and play' features and 'peer-to-peer' functionality.

- It adjusts to abnormal situations (unintentional islanding or faults).

- It uses local information to control the power flow of DG units.

- It can possess ac and/or dc distribution networks.

- It may provide electrical energy and thermal energy (hot and/or cold) simultaneously.

\subsection{Definitions of microgrids}

According to 79], a microgrid is a subsystem consisting of generation and associated loads that uses local control to facilitate its connection and disconnection to/from with the main grid in order to maintain a standard service during disturbances without harming the integrity of the transmission grid.

According to [84, a microgrid is a possible future energy system paradigm formed by the interconnection of small, modular generation units (micro-turbines, fuel cells, PV, etc.), storage devices (flywheels, energy capacitors and batteries) and controllable loads in LV distribution systems. Such systems can be operated interconnected to the power grid, or islanded, if disconnected from the grid.

In [85, a microgrid is defined as a cluster of distributed resource units and loads serviced by a distribution system which can operate in a 1) grid-connected mode, 2) islanded (autonomous) mode, and 3) a ride-through process between these two modes.

According to the U.S. Department of Energy Microgrid Exchange Group [86, a microgrid is a group of interconnected loads and distributed energy resources within clearly defined electrical boundaries that acts as 


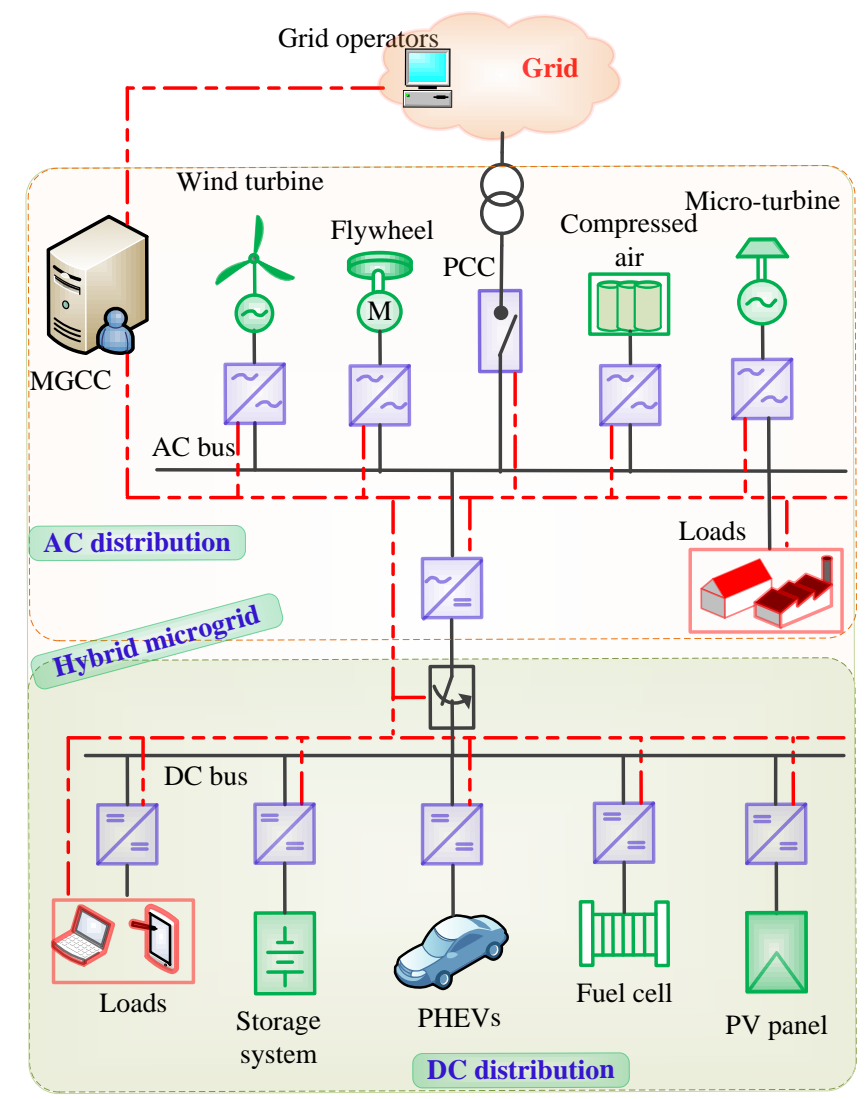

Figure 6: Hybrid microgrid.

a single controllable entity with respect to the grid. It can connect to and disconnect from the grid to enable it to operate in both grid-connected and islanded modes.

CIGRÈ C6.22 Working Group in Microgrid Evolution Roadmap 87] describes microgrids as electricity distribution systems containing loads and distributed energy resources (such as distributed generators, storage devices and controllable loads) that can be operated in a controlled, coordinated way either when connected to the main power network or islanded.

All the above definitions agree on the one point that a microgrid can be operated in either a grid-connected or an islanded mode in a defined area to act as a controllable entity. However, these definitions do not mention a control unit for facilitating the system's features, such as seamless transfer, and working as a supplementary power supply during an islanded operation. Also, they may not be appropriate for an islanded microgrid with converter-interfaced generators that need a central control unit to obtain second-to-second power balancing and optimal use of these sources.

Based on the above definitions and characteristics, in this paper, a microgrid is defined as follows. $A \mathrm{mi}-$ crogrid (consisting of small-scale emerging generators, loads, energy storage elements and a control unit) is a controlled small-scale power system that can be operated in an islanded and/or grid-connected mode in a defined area to facilitate the provision of supplementary power and/or maintain a standard service.

Its control unit, which is the main part of a microgrid, facilitates a systematic approach for obtaining a high penetration of RESs and reliable power supply through central regulation policies/algorithms. Although this approach basically distinguishes between a microgrid and distribution network, there are some confusing terms 


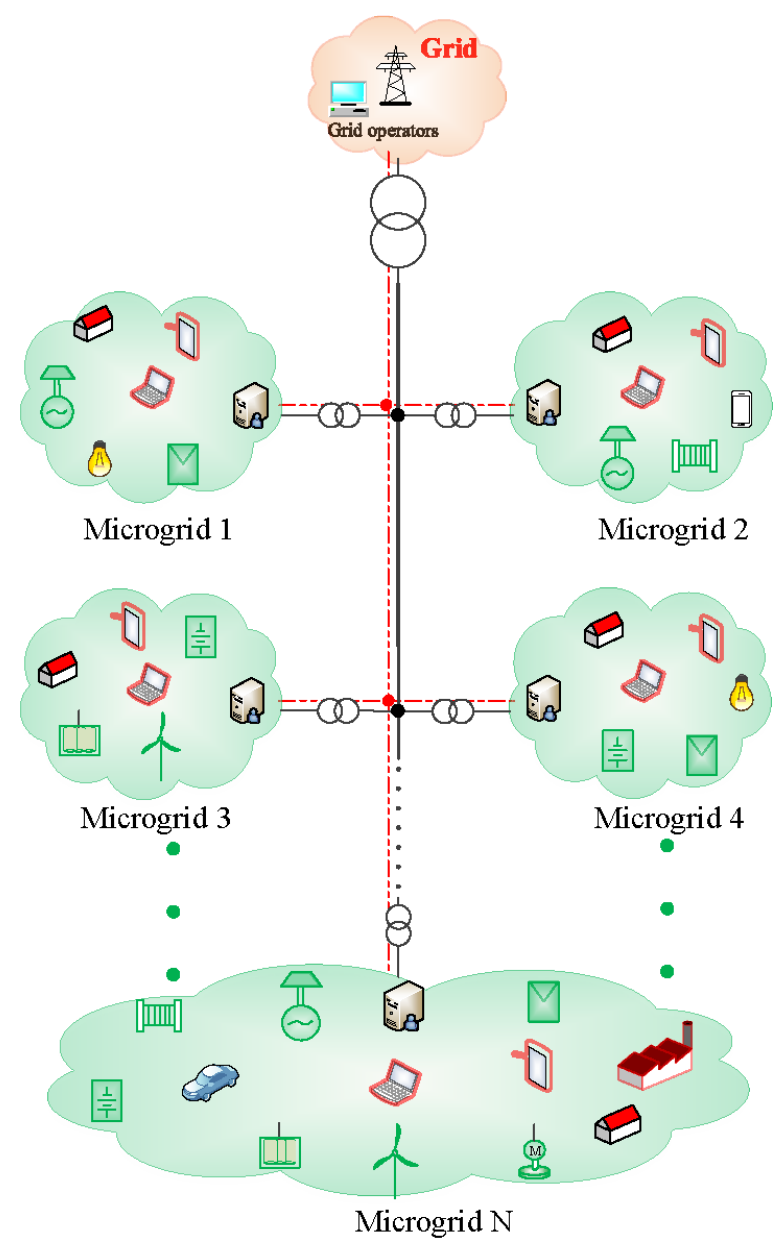

Figure 7: Concept of multi-microgrid.

regarding microgrids in the literature. One of these is a hybrid microgrid, which is often used in literature to indicate a combination of different types of DGs (diesel, PV, wind and battery) 91, 92, 93, 94. However, this cannot be considered as a hybrid microgrid because a microgrid itself consists of these DGs by definitions. Some other incorrect terms are CCHP microgrid, standalone PV microgrid, hybrid PV-CSP-LPG microgrid, hybrid photovoltaic-battery-hydropower microgrid, hybrid multi-microgrid and multi-bus microgrid system [95, 96. 97, 98, 99, 100. It is worth mentioning that the emerging active distribution network (ADN) has lots of microgrid features, such as power electronic devices including Soft Open Point (SOP) which are used to realise voltage and power regulation, and part of the distribution system can be operated in islanded mode, but it is not considered as a microgrid unless a central control unit is used.

Remark 1: The term 'and/or' is added to the microgrid definition to include all types of microgrids, for example, islanded and grid-tied ones. 'And' does not cover an islanded microgrid due to its lack of gridconnection facilities whereas 'or' refers to one mode of a microgrid operation, i.e., either grid-tied or islanded.

\subsection{Microgrid classification}

\subsubsection{Classification of microgrids based on power supply}

Microgrids are classified in three categories based on the characteristics of power they inject into a distribution network, as shown in Figure 8, and briefly described in the following subsections. 
Table 6: Comparison of ac and dc microgrids.

\begin{tabular}{|l|l|l|}
\hline Factors & AC & DC \\
\hline Conversion efficiency & Multiple energy conversions reduce efficiency & Less conversion processes increase efficiency \\
Transmission efficiency & Continuous reactive current loss reduces efficiency & Absence of reactive components increases efficiency \\
Stability & Affected by external disturbances & Free from external effects \\
Synchronisation & Synchronisation required & No synchronisation issues \\
Power supply reliability & Supply can be affected during seamless transfer & Power supply generally reliable \\
Microgrid controls & Control process complex due to frequency & Simple control approach \\
Protection system & Simple, cheap and mature protection schemes & Complex, costly and immature protection components \\
Suitability & AC loads & DC loads \\
Calculation methods & Complex numbers involved & Only real numbers used \\
\hline
\end{tabular}

AC microgrids: AC microgrids represent the ac power supply in a distribution network. They can be easily connected to an existing grid utility without special requirements such as converters and their control approaches. Since the evolution of the microgrid concept, ac microgrids have occupied a central place in research, with several publications regarding their control and operational approaches reported in the literature [101, 102, 103. This is because of well-established ac power networks with compatible modes of electrical and electronic devices. The distribution system of an ac microgrid can principally be classified as one of three types, single-phase or three-phase with/without neutral-point lines.

DC microgrids: The concept of a dc microgrid, which has a better short circuit protection with enhanced efficiency, has emerged due to the extensive application of modern electronic equipment and the availability of environmentally friendly dc sources (solar and fuel cells). These microgrids exhibit higher efficiency and have a lower conversion process for the application of dc loads than ac microgrids. Commercial applications (telecommunication systems, shipboard power systems and electric vehicles (EVs)) of an LV de distributed power system are reported in [104, 105, 106, 107]. The distribution network of a dc microgrid can be one of three types: monopolar; bipolar; or homopolar. The main difference between ac and dc microgrids is presented in Table 6 .

Hybrid microgrids: Hybrid microgrids consist of both ac and dc electricity distribution networks with an MGCC as shown in Figure6. The purposes of constructing hybrid microgrids are to minimise conversion stages, reduce interfacing devices, increase reliability and reduce energy costs, thereby improving the overall efficiency of the network. Such a structure enables both ac and dc power to be supplied to a distribution network while clients can use electricity according to their needs (ac or dc). Usually, PECs are responsible for decoupling the ac and dc parts of a microgrid [108, 109, 110].

\subsubsection{Classification of microgrids based on location}

Microgrids can be further classified based on their locations as urban or remote as shown in Figure 8 , brief descriptions of which are given below.

Urban microgrids: These microgrids are generally connected to a grid utility and, therefore, are capable of exchanging power with the grid through PCC. These microgrids, also known as the grid-connected microgrids, are capable of operating as an islanded microgrid during unusual situations, such as degraded power quality, faults on the main grid and the undertaking of network maintenance [4, 111. In these microgrids, all the rules and regulations, including complex control approaches, for the synchronisation process are satisfied to 


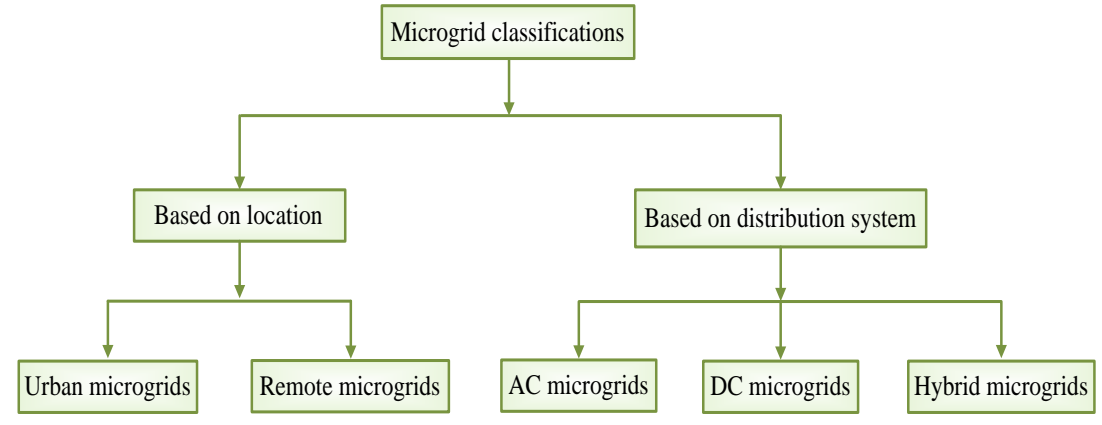

Figure 8: Classifications of microgrids.

maintain the power quality of the grid and preserve the microgrids' integrity for network stability. These types of microgrids can be constructed in both residential and commercial premises, such as university campuses, hospitals, data centres, communities, industries and shopping malls.

Remote microgrids: These microgrids are constructed in remote areas where a grid utility has no access for geographical reasons, such as military installations, islands and hilly areas [1, 112. As these microgrids, also known as islanded microgrids, are not connected to the grid supply, they do not require rules and regulations like urban microgrids. They are not as common as urban microgrids due to the lack of investment in them, and economic, political and technological reasons, with remote and island communities their prime locations.

\subsection{Challenges and opportunities of converter-based microgrids}

In a traditional power system, output power of a large generator can be controlled according to power demand, and the rotor of it are rotated at a constant speed, thereby obtaining a fixed network frequency. However, most DG technologies generate uncontrolled power with different voltage magnitudes and frequencies. As this process is quite different from that of the conventional power generation, it is challenging to integrate DG units in a distribution network. To achieve this, PECs play a vital role by minimising harmonics and generating the required voltage, frequency and power [113, 114, 115]. Although discussing all the microgrid issues in detail is beyond the scope of this study, a few recently published articles are presented to provide a comprehensive review of the overall challenges of microgrids.

\subsubsection{Challenges of microgrids}

A microgrid is different from a distribution network and has both additional advantages and challenges, with a major difference its application of an energy management system or centralised control unit in a defined region. In an islanded microgrid, which is not connected to a grid utility due to the geographical area, this control unit is used to maintain a network's optimal operation. However, in a grid-connected one, it has the additional requirements to protect against islanding, synchronise with the grid utility and disconnect the microgrid.

Microgrid central controller (MGCC): An MGCC is a physical computer system consisting of a software platform in which various modules for generation and load forecasts, human machine interfaces and supervisory control and data acquisition (SCADA) are used 116. It executes several processes for forecasting power generation, power demand and electricity market prices, and monitoring, analysing and optimising data, as shown in Figure 9, to provide the functions necessary for the effective operation of a power system by sending 
optimal decisions in order to ensure a power supply at the minimum cost. An MGCC can be realised for the both centralised and decentralised information processing. In a centralised processing, the central controller processes all the information of a microgrid while, in a decentralised controller, the central controller takes into account only a limited number of information with the real-time operation [117.

An MGCC is essential for coordinating a microgrid's different components in a systematic way to ensure its secure, reliable and economical operation. It enables a distribution network to integrate a high penetration of RESs and control them in an optimal way while the microgrid is either grid-connected or islanded. As its control approach is the key element of a microgrid, it needs to be designed in a way that represents the microgrid as a single entity from the grid perspective. Generally, a hierarchical control approach, in which primary, secondary and tertiary control levels are embedded, is responsible for its coordination [4, 118, 119]. The role of the primary control level is to ensure proper power sharing among DG units and control the terminal voltage while those of the secondary and tertiary ones enable optimal operation of the microgrid considering multiple objectives. Conventionally, these three levels, which incur a heavy computational burden and considerable costs while providing low reliability due to a single point of failure, send necessary signals through low-bandwidth communication lines to all the DG units. However, due to the development of advanced control approaches, the primary and secondary control levels can be implemented in decentralised ways, e.g., in a local DG unit controller (Figure 10), to enhance a microgrid's reliability during a single point of failure.

To avoid expensive communication lines and increase the reliability of power sharing, the primary control level generally adopts the droop control concept and the secondary one a MAS in a distributed manner to restore the voltage and frequency [120, 121]. In MAS technologies, local controllers can make decisions autonomously regarding controlling their own DG units by communicating with neighbouring ones. A distributed peer-to-peer MAS for improving power sharing among DG units in a microgrid is demonstrated in [120]. An ontology-driven MAS for implementing the energy management of microgrids considering DG units and non-critical loads is presented in [122]. A review of MAS technologies used for microgrid control and optimisation is provided in 8. Although a MAS framework can work in a grid-connected microgrid, it may not do so effectively during an islanded operation.

Although the primary and secondary control levels can be implemented in a decentralised manner to increase the reliability of a microgrid, it is important to have a centralised tertiary control level for each microgrid to coordinate it with other microgrids and the grid utility as well as provide optimal energy management of DG units in the presence of a high penetration of RESs [123. An MGCC detects the power quality at the PCC to determine whether to operate in an islanded or grid-connected mode and can re-synchronise an islanded microgrid with the grid utility once the grid restores the appropriate power quality [124, 125]. As the different features of the energy sources in a microgrid produce fluctuating power levels which create challenges for an MGCC to balance the power in a network, various control methods for an MGCC are proposed in the literature [126, 127, 128, 129. Balancing the power to avoid voltage fluctuations is an essential task of a microgrid for its safe and reliable operation. An autonomous load-curtailment algorithm for disconnecting loads during peak-load hours to minimise the power difference between supply and demand is developed in [128. An algorithm which optimises the power generation of a microgrid and its exchange of power with the grid utility is proposed in [127. As energy storage units are responsible for regulating the voltage's amplitude and frequency in an islanded microgrid, an MGCC approach for the microgrid's safe operation within their 


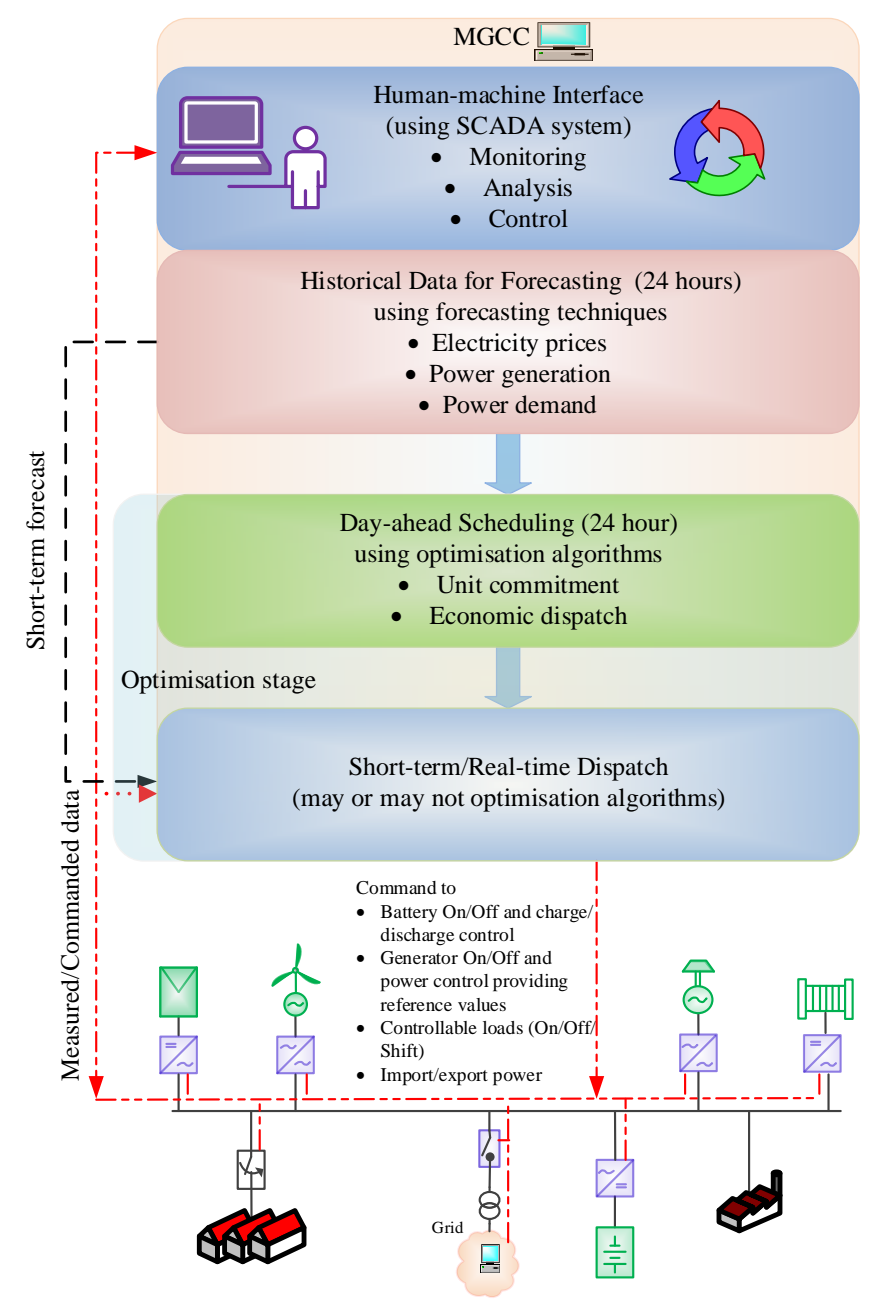

Figure 9: Typical stages of supervisory controller.

limits to prevent its fast degradation or damage is proposed in [129. This method avoids the overcharging of these units by curtailing their generation of active power, their deep discharging through load shedding and their uneven degradation through equalising the charge state among the units.

Microgrid stability: In a conventional power system, stability problems that define the ability of a power system to maintain synchronism during disturbances are categorised into rotor angle stability, voltage stability and frequency stability [55]. While rotor-angle stability provides information regarding the balance between the electro-magnetic and mechanical torques of a generator rotor required for it to remain in synchronism, voltage and frequency stabilities refer to the balance of a network's power supply and demand. Although the dynamic behaviour of a synchronous generator has a substantial role in maintaining power system stability, in a microgrid with converter-interfaced DGs, rotor angle stability is no longer an issue due to the lack of inertia of DGs that makes a significant difference in operating a microgrid from the traditional grid [1]. In addition, a microgrid also suffers from the buffer energy of an inter-connected transmission system where energy is stored in the inductance and capacitance of line parameters. Although the voltage and frequency stabilities in a microgrid are analysed in an islanded mode, only the former is controlled in a grid-tied operation due to the small effect of the frequency on the grid [7] because the voltage acts as a local variable and the frequency as a global one. Both stabilities are determined through either a small- or large-disturbance analysis, with the 


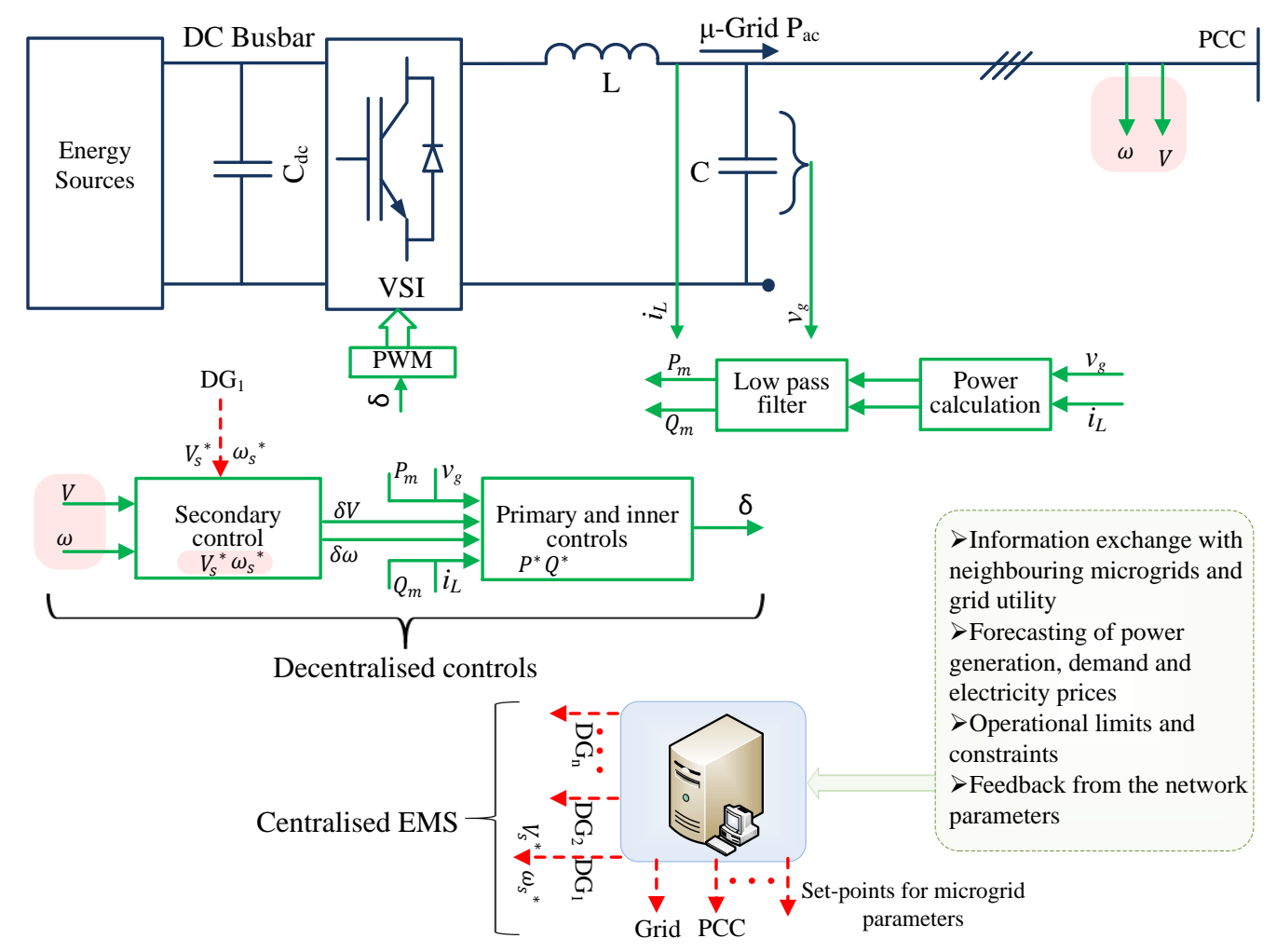

Figure 10: Centralised and decentralised controls of microgrids.

stability of a microgrid basically dependent on its operational modes, control topology, types of DGs and loads, and network parameters [130, 131, 132].

A small-disturbance analysis involves evaluating a microgrid's stability in terms of linearising the system around its operating points using eigenvalue analysis, Routh-Hurwitz or Nyquist stability criteria. Several small-signal analyses that deal with load fluctuations, and the influence of droop gains and variations in the line impedance on voltage and frequency characteristics are conducted in 130, 133, 134, 131, 135, 61, 62. As droop coefficients have a direct influence on a microgrid's small-signal stability, accurate design of droop coefficients and a droop control with adjustable dynamic behaviour are recommended in [61, 62, 136]. A smallsignal analysis of a grid-connected inverter for use in an UPS unit is first demonstrated in [137. Then, in [61, inverter-based microgrids with linear loads are modelled to perform a small-signal analysis of the frequency and damping of oscillatory components. To demonstrate the influence of an active load in a microgrid, in [62, a network is modelled with non-linear loads and it is found that the low-frequency modes are linked to the voltage controller of the active rectifier and the large load gains cause instability in the inverter's operation. In [63], a synchronous generator is added to an inverter-based microgrid to investigate the stability margin, with a modified droop control presented. A reduced-order model using the Kron reduction method for inter-inverter oscillations is developed in [138. In [139], inertia constant and frequency droop coefficients are tuned together by applying the knee-point-driven evolutionary algorithm (KnEA) to enhance the stability of the frequency of a microgrid. The main disadvantage of a small-signal analysis is that it is only effective around the linearised operating points and does not indicate the ranges. However, this can be overcome by implementing non-linear techniques such as Lyapunov methods. To achieve improved performances, researchers can still obtain an accurate model of a microgrid and select optimal control gains using advanced optimisation algorithms. 
A large-disturbance stability analysis determines a system's capability to retain a network's stability after a a microgrid to improve stability. An overview of a microgrid's stability issues and mitigation procedures is presented in Figure 11. It is worth mentioning that a microgrid's stability is divided into mainly frequency and voltage stabilities. The small- and large-disturbance stability are categorised as analysing methods not the types of stabilities reported in [7, 147. While the majority of research presented to date is based on radial

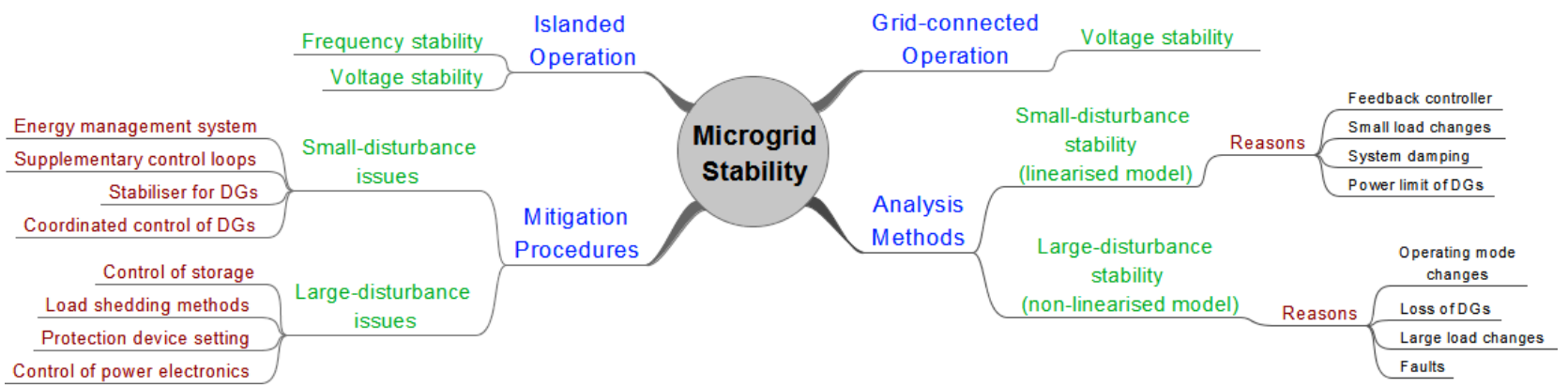

Figure 11: Pictorial overview of microgrid's stability issues and mitigation procedures.

networks, studies of the stability of mesh ones are still needed

Protection: A conventional distribution network, the current flow of which is unidirectional, is equipped with protection devices, such as reclosers, circuit breakers, fuses and relays, to ensure fault detection and isolation, with the former generally carried out using over-current relays. However, integrating DG units in 
a radial network makes the network a complicated one due to the difficulty of analysing the direction and magnitude of the bidirectional current flow [152. In addition, DG units in a microgrid need to be protected in both grid-connected and islanded operations, where a fault current changes significantly for altering the modes. The reason is that the utility supports a huge flow current in a grid-tied microgrid, whereas converter-interfaced DG units limit the fault current during an islanded operation due to stochastic nature of power generation and power limits of electronic components [153. This limit may be so low that conventional protection systems, such as fuses, over-current relays and reclosers, may not accurately detect faults due to their pre-set current limits and, consequently, may be inadequate for completely protecting a microgrid. A fault current is affected by the capacities, types and locations of DG units which may cause miscoordination among primary backup relays and lead to the false tripping of protection devices, unintentional islanding, failure of auto-reclosing and fault detection [154]. Therefore, it is essential to design a proper protection scheme that can be operated under different microgrid conditions.

The microgrid protection system can be classified into two types, 1) a static switch at the PCC of the microgrid to first isolate the microgrid from the distribution network in spite of internal or external faults of the microgrid; 2) the microgrid protection system which contains two different individual protection functions, one for the grid-connected operation mode and the other one for the islanded operation mode. Several protection methods for solving protection issues are proposed in the literature. One is changing the protective devices or settings by re-calculating them according to changes in the network's topology [155, 156, 157, 158]. Its main drawback is that it does not consider an increasing penetration of DG units, uncertainties inherent in power generation and demand, and reconfiguration of the network [159. A fault current limiter (FCL), in which a limiter (impedance) is connected in a series with DG units, is another approach for minimising the effects of the units on the protection system [160, 161, 162. In a normal operation, the value of impedance is almost zero but rises and limits the short-circuit current when a fault is detected. Although this method can reduce the negative impact of installing DG units, it is only applicable for specific types of microgrid topologies due to its expense and need to select the best location for the series impedance for every microgrid configuration.

To overcome the above issues, an adaptive protection scheme which changes the parameters of protective devices via communication or measurements in response to a change in a microgrid's configuration is presented in [163]. It constantly monitors the system's status using a communication channel to update the coordination of its protection devices during changes in its operations or topology. It consists of directional over-current relays with a changeable group of settings and high-speed, secure communication channels. It can be operated in both a centralised and decentralised manner whereby a microgrid's protective settings are updated either online or offline for each change in its topology [164]. In an offline process, all possible settings for different scenarios in a microgrid's grid-tied and islanded modes are first stored in a memory of a supervisory remote control unit (SRCU), then it analyses the microgrid's status to decide the settings of online relays for any change in the network's topology [165]. However, an offline method may not work for all dynamic situations and simultaneous faults due to a large number of conditions [166].

A SRCU is used in a centralised adaptive protection scheme to adjust relay settings to obtain an appropriate current level via a high-speed, secure communicative infrastructure while a decentralised one, in which a set of distribution agents of various devices is operated in a coordinated way through communication channels, has a higher speed, and greater reliability and expansibility [166, 167]. Although adaptive protection is an effective 
Table 7: Comparison of islanding detection methods [170 179].

\begin{tabular}{|l|l|l|l|l|l|}
\hline Features & Active & Passive & Hybrid & Remote \\
\hline Operation Principle & Injecting disturbance signal to grid & Monitoring PCC parameters & Combination of active and passive & Communication between DG & Extraction of features \\
NDZ & Small & Large & Very small & Zero \\
Response time & Very short & Short & Longer than active methods & High \\
Nuisance tripping percentage & Low & High & Lower than active and passive & Negligible \\
Applicability in multiple DG system & Not preferable & Highly preferable & Not preferable & Preferable \\
Implementation cost & Minimum cost & Average cost & High cost & Low \\
Effect on microgrid & Greatly decreases power quality & None & Degrades power quality & None \\
\hline
\end{tabular}

approach for both the operating modes of a microgrid, outages of communication channels and cyber security threats are its major drawbacks. In [168, an adaptive protection scheme considering the existing ESSs in a network is designed to increase resiliency against communication failures. Further analysis aimed at designing a robust adaptive protection scheme that can efficiently respond in terms of uncertainty in both modes of a microgrid operation is still needed.

Island detection of microgrids: The protection scheme in a traditional power system is designed based on the assumption that, in the case of a fault or an unwanted event, the distribution system will be isolated from the network. However, for a DG-integrated network, the system remains energised in the event of a fault, with the loads supplied from local DG sources and its operation under such a condition referred to as that of an islanded microgrid [169]. The main reasons for islanding include switching operations, such as clearing faults, shedding unexpected large loads and maintaining outages [170, 171].

There are two islanding operations: intentional; and unintentional. The former, which is an action conducted by grid operators, is primarily executed to either implement a power management plan or avoid cascaded failures. Due to its systematic procedure, it is generally safe and can also be implemented to improve the power quality of a microgrid [170. In contrast, an unintentional islanding is an unexpected operation of a microgrid. If an unintentional islanding condition is not detected precisely in a timely fashion, the entire network may collapse with several negative effects, such as the introduction of safety hazards for operational personnel, damage to customers' equipment, voltage and frequency instabilities, and problems while re-closing the converters [172. For these reasons, current standards, such as IEC 62116, IEEE 929-2000 and IEEE 1547, do not permit DG units to be operated in an islanded mode $[173,171$. However, this is not economically viable as local resources may fully or only partially fulfil the power demand without interrupting a continuous power supply. In order to enable a safe microgrid operation, researchers are continuously working to develop efficient detection techniques by addressing different technical issues.

Although detecting unintentional islanding conditions is challenging, achieving this as soon as possible is essential in order to take immediate action to establish a stable microgrid operation. According to IEEE Standard 1547-2003, the maximum permissible time for detecting islanding conditions is 2 seconds [174. Several methods in the literature based on electrical quantities, such as voltage, frequency, impedance and power, for effectively detecting islanding conditions $[169,172,175,176$, are mainly classified as remote, local and intelligent [177.

Remote detection techniques are based on expensive communication lines between a utility and its DG units [170, 178. In them, power-line carrier communication (PLCC) and SCADA are used to execute an islanding operation [170. Although they provide better reliability for multiple DG units than local methods, they are often criticised for the high expense involved in installing sensors and communication devices. 
Local techniques widely used to detect islanding are classified based on their measurements of electrical quantities (voltage, current, frequency and harmonic distortion) at DG sites as active, passive and hybrid. The active ones deal with changes in the small signals injected into a grid to detect abnormalities in its operation while passive ones monitor the electrical quantities at the PCC to observe the transient phenomena in the network [180. The hybrid method consists of both active and passive techniques, with the latter used first to detect islanding and then the former applied. It has lower non-detection zones $(\mathrm{NDZs})$ - the zones of failure for detecting islanding situations on time - and less impact on power quality. Although passive methods are cost-effective and simple to implement, they may fail to accurately detect islanding conditions due to large NDZs. To overcome this limitation, active methods using a small NDZ are employed but require extra time to respond to perturbations [181, 170].

Several approaches based on intelligence techniques, such as wavelet analysis, and neuro-fuzzy and adaptive artificial neural networks (NNs), for effectively detecting and classifying islanding conditions have recently been developed [182, 183]. Their signal-processing techniques facilitate the extraction of hidden characteristics of the relevant network's conditions, such as faults, power quality and maintenance, with any transfer between these modes required to be seamless and separating a microgrid when a grid fault occurs should not affect the loads. According to IEEE-1547-2003, an anti-islanding scheme must de-energise the sources within 2 seconds of an islanded operation due to concerns about stability and protection issues [184] while, according to IEEEthe criteria of adequate levels of voltage and frequency stability [185. However, when switching from a current controller mode in a grid-tied microgrid to a voltage controller mode in an islanded one, the outputs from these controllers may not be equivalent at the exchanging moment and lead to current or voltage spikes at that instant [186, 187. In contrast to the re-synchronisation of a conventional power system, where a synchroniser acilitates the process for a single machine, a microgrid is quite different due to its multiple DGs with different dynamic attributes and should restore the grid quickly and smoothly to avoid any disturbance in the power supply to loads [188]. The main difficulty for re-synchronisation is to precisely recognise the moment at which the static switch at the PCC is closed for a specific voltage, frequency and phase of the utility grid and that of the microgrid to avoid circulating current flows in the network. An inappropriate selection of this instant can lead to a circular current, subject to the momentary voltage distinction between the grid utility and microgrid, that may conceivably harm the system's components or falsely trip its protection devices [186].

It is important to constantly monitor the voltage, frequency and phase of a grid utility for its correct operation and re-synchronisation with an islanded microgrid. However, the synchronisation criteria presented in IEEE Standard 1547-2003 may not be appropriate for a converter-based microgrid which has a low inertia, a re-synchronisation process depending on the different dynamic sources. In the first, initially, all the sources 
are synchronised simultaneously to construct a microgrid bus which is then synchronised with the grid. The second is to synchronise one of the DGs with the grid first and then the others consecutively [188]. In the former approach, it is difficult to gain a grid voltage template for every DG scattered over an extensive region while, in the latter, the time required for the synchronisation process is much longer and leads to poor resource usage.

In order to minimise the transient effect of electrical parameters during mode changes, a method of seamless transfer, in which the voltage and current controllers are interchangeably used for different purposes in both operations by readjusting their reference values, is presented in [186] but the time interval during the switching process is neglected. An intelligent load-shedding scheme with an algorithm for intentional islanding and reconnection, which reduces reliability due to its load-shedding techniques, is developed in [190]. A droop-based approach, in which one DG is designed as a dispatchable unit for facilitating a smooth transition between two modes, supplies extra power immediately after the loss of the main grid for the transition and remains idle for most of the time, is implemented in 191. A hierarchical architecture with primary and secondary control levels that considers power quality issues for achieving a seamless transitions is designed in [187. It allows transitions to be performed without disconnecting the DGs or loads but does not consider a backup generator, apart from an ESS. The techniques discussed above are mainly applicable for UPS systems as they do not consider the dynamics of RESs.

To facilitate re-synchronisation, three types of strategies are reported in [192, 184]: (1) active synchronisation in which a central control technique coordinates the voltage, frequency and phase angle of an islanded microgrid with the grid using communication lines [126, 193, 194]; (2) passive synchronisation in which a synchroniser checks for parallel devices using a synchrocheck relay [195, 196]; and (3) open-transition transfer synchronisation in which DGs and loads are de-energised before their re-connection. The third strategy has reliability issues due to its discontinuous power supply to loads during transitions, the second, although simple because of the availability of well-established synchrocheck relays in practice, may suffer from poor voltage and frequency control 192 while the first is suitable for converter-based microgrids due to its communication facilities. In [126, an active synchronisation control scheme which uses network-based control of multiple DG units to set the voltage and frequency of a microgrid is demonstrated. It permits the synchronisation of a microgrid under different working states of DGs and load changes but doesn't accomplish a quick and optimised synchronisation. This work is extended by designing a robust controller based on a $\mu$ synthesis analysis in [197. An approach for synchronising the frequencies and phase angles of all DGs based on a combination of three distinctive phaselocked loops (PLLs) is demonstrated in [198. In these methods, a central control unit used to make decisions sends information via communication channels that are vulnerable for a large system during communication failures. To overcome such a concern, a combination of droop control, distributed cooperation control and an active synchronisation strategy for improvimg flexibility and redundancy is developed in [197. A bi-level distributed synchronisation control framework using minimum, sparse communication to adjust the output power of all DGs in order to regulate the voltage and frequency of a microgrid and track the grid components is presented in 199. A linear quadratic regulator-based bumpless transfer controller for the seamless transfer of a microgrid described in 200 reduces voltage and frequency oscillations during a transition. Control approaches based on observer and auxiliary tracking for transferring from power tracking or voltage control to voltage tracking or backup droop-based control, respectively, are developed in [201]. The research conducted to date 
on seamless transfers can be further extended by applying new approaches to ensure a smooth transition considering the presence of uncertainties and harmonics in a network.

Location and sizing of DG units in microgrids: Traditional distribution networks are radial in nature, whereby power flows from a distribution substation to consumers. However as, in a microgrid, DG units located in single or three phases close to the consumer end cause a bidirectional power flow, placing them in suitable places and determining their proper sizes are critical tasks [202] as inappropriate placements may lead to a microgrid being in an unbalanced condition and cause a reverse power flow and increased line losses. A greater penetration of single-phase DG units in a three-phase system increases the power loss and fault current 203. while their inappropriate placements also negatively affect the voltage quality and create harmonics in a microgrid [204]. Large DG resources alter a fault current's direction and phase angle, leading to the unwanted operation of protection devices. These issues can be overcome by the optimal planning of DG units and solving the appropriate objective functions and constraints using suitable algorithms. Most of the problems in early research studies are formulated based on a single objective, such as the minimisation of energy losses or total cost, for different perspectives of DG promoters or distribution network operators (DNO) [205, 206]. Although determining a solution of a single-objective function may create conflicts of interest among agents (DG promoters, regulators and DNOs), this can be minimised using a multi-objective function that provides a compromise.

Generally, objective functions are formulated as active power losses and voltage profiles for obtaining the types, numbers, sizings and sitings of DG units [207, 208, 209, 210. There are also other objective functions, such as reactive power minimisation, DG capacity maximisation, environmental cost reduction, reliability improvement, imported energy minimisation and spinning reserve power [211, 212, 213]. Feasible solutions to objective functions are often limited to imposing constraints on the power balance, line current, node voltage and power factor of a DG. The constraints can be further extended to the utility limitations, such as the power exchange, short-circuit current, transformer capacity, number of DGs and maximum power production of DGs [214.

Objective functions with constraints can be solved by applying various computational methods, such as classical and artificial intelligence ones. Classical approaches, which are based on determining a mathematical solution to a problem, include linear/nonlinear programming, dynamic programming (DP) and optimal power flow, while artificial intelligence ones, which are inspired by natural behaviour, include simulated annealing, harmony search, evolutionary algorithm, ant colony and hybrid intelligence (genetic algorithm (GA) - tabu search) techniques. Although an analytical approache is typically simple and highly precise depending on the model's accuracy, it may not be suitable for a complex system's multi-objective functions [215]. In this case, an artificial intelligence algorithm can be the best option due to its flexibility regarding these functions. However, 10 the solutions obtained by these methods may become trapped in a local minimum without reaching the global optimum. As a hybrid optimisation algorithm can overcome the difficulties of an individual one, it can be more suitable for the optimal selection of DG units as either discrete or continuous variables.

A mixed algorithm consisting of a GA and intelligent water drops (IWD) for determining the best locations and capacities of DG units, respectively, to minimise power losses, enhance voltage stability and control a microgrid is developed in [216. In [217, a new index for calculating the optimal locations and sizes of DG units considering load variations is presented. A hybridisation of heuristic search and analytical approaches, in which 
the sizes of DG units are determined by analytical methods and their locations by PSO, for optimal multiple DG placements is described in [218]. A multi-objective optimisation method for determining the optimal sites and sizes of multiple DG units is presented in [219. Further research in this area can be conducted by considering uncertainties in loads and RESs, and the optimal power factor and reactive power limits of DG units without violating constraints.

Locations and sizes of ESSs: A battery energy system is a type of ESS that has drawn significant attention for implementation in a microgrid due to its quick response, flexible application and easy control 220. It has a wide range of both economic and technical uses in a microgrid, such as minimising operational costs, reducing curtailed energy, minimising energy losses, improving network stability, shifting loads, supplying uninterrupted power and deferring the upgrading of transmission lines [221]. An ESS, a key element of a microgrid that helps to distinguish it from a distribution network, enables a microgrid to be operated in a stable islanded mode which is not possible for a distribution network, even with a high penetration of intermittent RESs. However, its initial cost is still a major concern that needs to be overcome before its potential advantages can be realised. Therefore, as there is a trade-off between obtaining an improved network performance and profitable investment, determining a battery's optimal size is required which is accomplished by applying computational techniques for various installation purposes, with the criteria primarily categorised as economic, technical and techno-economic 222. The economic deals with minimising the overall cost of installing an ESS in a system while the technical concerns about improving a network's dynamics by regulating the voltage and frequency of a microgrid. The techno-economic considers both the economic and technical perspectives, where the main goal is to reduce the overall cost while taking account of technical constraints. In addition, the lifetime of a battery should be considered when deciding on its optimal size in order to prolong its storage services.

The issues regarding determining a battery's capacity can be resolved using several techniques, each of which has its own advantages and shortcomings. The common methods applied in the literature are probabilistic, analytical, mathematical optimisation, heuristic and hybrid. The key idea in a probabilistic approach is to use the stochastic behaviours of RESs to better determine the optimal battery size. Although it can provide satisfactory results with little resource data, it can only handle a limited number of performance criteria 222. An analytical method, also known as a deterministic approach, is one of the most extensively used for determining ESS sizes. It is effective and flexible in terms of all the criteria and simulation methods in many cases but requires a large number of simulations which is problematic for limited computational resources $[223,224$. Although a mathematical optimisation algorithm can improve the computational efficiency of analytical approaches, it has convergence issues in the presence of non-linear complex problems. This has led to the introduction of heuristic approaches for finding near-optimal solutions rather than accurate results. The main strength of a heuristic approach is to avoid mathematical derivatives and thereby use reasonable amounts of memory and time 225. However, as there is a possibility of a heuristic method becoming stuck in a local optimum rather than a global one, using a combination of the above methods which overcomes their individual limitations could guarantee convergence toward global optimisation.

In 226, a cost-benefit analysis of the optimal sizes of ESSs for both grid-connected and islanded modes is demonstrated by applying a mixed linear integer problem (MLIP). An improved bat algorithm for determining the optimal ESS size of an LV grid-connected microgrid considering some constraints, such as the amount of DG power, ESSs' energy capacities, the operating reserve and load demand, is applied in [227]. An effective 
battery size for regulating the voltage and peak load under a high level of PV penetration is determined in 228. This study conducts a cost-benefit analysis that considers a battery's influence on the work stress of voltage regulation devices, load shifting and peak power generation, and the cost for its estimated lifetime. In [229, a probabilistic model for obtaining the optimal sizing and siting of a battery system in a distribution system is developed considering both the operating modes of a microgrid for minimising the amounts of energy storage, energy losses and expected energy not supplied. A multi-objective sensitivity algorithm for optimising the capacities of PV and storage systems is presented in [230. As a power-flow algorithm is used to control the reactive power flow of a solar system, voltage variations are minimised, energy savings maximised and peak loads reduced. Different architectures, sizing methods, design criteria and control techniques for ESSs in microgrids considering relevant constraints are discussed in [231. Taking different objective functions with constraints into account, the work can still be advanced by the application of novel optimisation algorithms.

Power sharing: A basic control approach for converter-based DG units is adopted from the control for an UPS unit which is similar to an islanded microgrid. As a microgrid consists of multiple generators, unlike a stand-alone UPS that generally uses a single storage or generating unit, special control strategies are needed for accurate sharing of the generated power (active and reactive) among its DG units. This can ensure the stability, reliability and economical operation of a microgrid during load changes and depends greatly on the types of energy sources, i.e., PV, wind, micro-turbine and fuel cells, distribution lines and non-linear loads. As power sharing is challenging due to the uncertainties inherent in the power generated by RESs and the resistive nature of distribution lines 232, proper strategies for load distribution need to be improved to increase a microgrid's reliability. Power sharing can generally be performed in one of two ways, communication-based or communication-less.

Communication-based power control achieves effective power sharing and voltage regulation at the expense of a high investment cost for the communication's infrastructure. These methods include centralised, masterslave, average load sharing, peak value-based current sharing, circular chain and distributed forms of control [4] and, of them, a master-slave control approach is widely used in practice [233, 234]. In it, the master converter that is in charge of a microgrid control works as a VSC by producing a controlled voltage and frequency while slave converters act as CSCs by obeying the current pattern ordered by the master inverter in order to achieve effective power sharing among DG units [235]. Although a master-slave method can achieve good power sharing with the benefit of easy installation, its expensive communication lines reduce the reliability of a microgrid during communication failures or delays as well as limit any increase in the number of DG units 44.

Communication-less power sharing based on droop characteristics that avoids expensive communication infrastructures and increases a system's reliability and redundancy requirements is proposed in the literature. It is particularly helpful for connecting remote DG units and can be easily expanded due to its plug-and-play features that permit one unit to be replaced without stopping the entire system [4, 236]. A drop in the voltage and frequency at a DG's terminal during any disturbance is the key concept of droop control which facilitates the plug-and-play feature without depending on central commands. The main advantages of droop control methods are their better flexibility and reliability than other techniques. However, their disadvantages are their coupling of power control, dependence on network impedance, issues with non-linear loads, and voltage and frequency deviations 4. To overcome these problems, different control approaches together with their relative strengths and weaknesses are discussed in the literature. 
In [237, a virtual flux method for improving frequency deviations by reducing the multi-feedback loops of an inverter control is presented. A voltage/current (V/I) droop-based control approach for enhancing the reactive power sharing, dynamics and stability of a microgrid is proposed in [238]. Voltage-based droop control, which includes the characteristics of RESs in the power-sharing strategies of a microgrid, is described in 239. To minimise power-coupling issues due to the near-unity ratio of $\mathrm{R} / \mathrm{X}$, modified droop control, virtual inertia and universal droop control methods are presented in [240, 241, 242, 243]. Different methods for the power sharing of DG units is discussed in [4, 244, with their relative benefits and drawbacks shown in Table 8 , Researchers can still improve the power sharing strategies by employing new control strategies and optimisation algorithms that can tune the co-efficiency of the controllers for better performance.

Table 8: Benefits and drawbacks of power sharing strategies.

\begin{tabular}{|c|c|c|}
\hline Control Methods & Advantages & Disadvantages \\
\hline Communication-based methods & $\begin{array}{l}\text { Accurate power sharing and voltage regulation } \\
\text { Improved transient response } \\
\text { Elimination of circulation currents }\end{array}$ & $\begin{array}{l}\text { Expensive communication lines } \\
\text { Microgrid reliability issues } \\
\text { DG expansion and flexibility issues }\end{array}$ \\
\hline$P / f$ droop & $\begin{array}{l}\text { Suitable for high and medium voltage line } \\
\text { Not dependable on communication line } \\
\text { Easy implementation and flexible expansion }\end{array}$ & $\begin{array}{l}\text { Sluggish dynamic response } \\
\text { Poor reactive power regulation } \\
\text { Sensitive to physical components }\end{array}$ \\
\hline$P / V$ droop & $\begin{array}{l}\text { Suitable for low voltage distribution line } \\
\text { Not dependent on communication line } \\
\text { Easy implementation and flexible expansion }\end{array}$ & $\begin{array}{l}\text { Sluggish dynamic response } \\
\text { Poor active power regulation } \\
\text { Sensitive to physical components }\end{array}$ \\
\hline Adaptive derivation & $\begin{array}{l}\text { Enhanced power sharing } \\
\text { Eliminates voltage and frequency distortions } \\
\text { Improves dynamic stability of power sharing }\end{array}$ & $\begin{array}{l}\text { Not suitable for complex network } \\
\text { Difficult to implement multiple DGs }\end{array}$ \\
\hline Frequency based signal injection & $\begin{array}{l}\text { Suitable for different types of load application } \\
\text { Robustness in system parameter variations }\end{array}$ & $\begin{array}{l}\text { Harmonic issues in voltage control } \\
\text { Complex implementation }\end{array}$ \\
\hline Voltage based droop & $\begin{array}{l}\text { Suitable for highly resistive network } \\
\text { Suitable for renewable energy control } \\
\text { Easy power balancing }\end{array}$ & $\begin{array}{l}\text { Difficult practical implementation } \\
\text { Voltage varies during load changes }\end{array}$ \\
\hline Virtual flux control & $\begin{array}{l}\text { Improved frequency control } \\
\text { Simple control structure }\end{array}$ & $\begin{array}{l}\text { Difficult to implement in a large system } \\
\text { Slow dynamic performance }\end{array}$ \\
\hline$V / I$ droop control & $\begin{array}{l}\text { Improved faster dynamic response } \\
\text { Ensures accurate real and reactive power sharing } \\
\text { Suitable for small-inertia DG units }\end{array}$ & $\begin{array}{l}\text { Oscillation issue for small droop coefficients } \\
\text { Voltage issue under heavy load conditions }\end{array}$ \\
\hline
\end{tabular}

Microgrid planning: The problem of a microgrid planning, which involves determining the economic viability and optimal selection of a microgrid's parameters before its practical implementation, is a challenging task because of the involvement of uncertain data, such as forecasting errors in loads, renewable generations and market prices, during assessments of the microgrid's deployments 245, 246. Therefore, because of a microgrid's high cost, efficient planning techniques need to be developed to ensure a profit and justify further investment based on cost analyses of unpredictable situations. In [246, an economical model that considers uncertainties in physical and financial information is developed for efficient and viable microgrid planning. A theoretical framework, including a long-term investment and short-term cost, for studying the cooperative planning of RESs in a multi-microgrid operation is presented in [247]. It is observed that, compared with non-cooperative microgrids, these cooperative ones obtain a $35.9 \%$ reduction in a system's overall cost. Two-stage stochastic 
programming for minimising the risk and expected cost of an investment that considers different scenarios of uncertainty over the data is described in [248. It is concluded that risk-averse stochastic programming obtains better results in terms of economic benefits than deterministic approaches while still satisfying environmental objectives. In 249, the optimal planning of a multi-microgrid considering different factors, such as network- and resource-based uncertainties, to increase reliability and operate economically is investigated using a probabilistic minimal cut-set-based iterative methodology. A robust optimisation approach considering economic, technical and reliability aspects for the optimum design of a microgrid and employment of DG units with a section switch and tie switch allocation is presented in [245. In 250, a tool for the long-term planning (up to 20 years) of an industrial microgrid taking into account the objectives of all the stakeholders (prosumers, consumers and the microgrid's energy manager) is developed using a game theoretical framework.

Forecasting data: The intermittent nature of RESs together with the unpredictable participation of loads poses great difficulties for balancing power generation and demand. To address these challenges, a spinning reserve becomes an essential part of a microgrid as an energy buffer to relieve fluctuations. However, it increases the monetary and related administration costs which lead to less motivation for investing in a microgrid-related infrastructure [251. Therefore, to obtain precise estimations of the levels of power generation and demand, from a few minutes to a few days, a forecasting method for scheduling a microgrid's devices, as shown in Figure 9. is essential for achieving a cost-effective and reliable operation. This is a challenging task because of the uncertainties in RESs, loads and real-time electricity prices. Prediction periods can be divided into various time horizons, such as very short-term $(1 \mathrm{~min}-1 \mathrm{~h})$, short-term $(1 \mathrm{~h}-1$ week $)$, medium-term $(1 \mathrm{month}-1$ year), and long-term (1 year and above) [252. Very short-term forecasting is used for real-time power dispatch, optimal reserves and power smoothing while short-term forecasting ensures the unit commitment and economic power dispatch/scheduling. Medium-term forecasting methods are used to plan and maintain a power system by estimating its future available power supply and long-term ones in the planning phase of power generation, transmission and distribution systems to determine the appropriateness of the investment 253 .

Forecasting techniques can be generally categorised as statistical, machine learning, physical/numerical and hybrid. Statistical ones depend on analysing historical data in a time series of the variables, where input data are decomposed into time series, a regression of which is used to estimate future specific variables. Common statistical methods include linear regression, autoregressive moving average (ARMA), autoregression with exogenous inputs (ARX), sparse vector autoregression (sVAR) and Grey models [251, 252]. Although a statistical method is computationally efficient, a machine-learning technique, such as a neural network (NN), is suitable for the interpretation and prediction of non-linear relationships related to historical data. In it, a $\mathrm{NN}$ is first trained using approximately $70 \%$ of the total amount of data with the help of a back-propagation algorithm, and then tested using the remaining $30 \%$. The main drawbacks of a NN are its requirement for a large amount of data and its over-fitting issues during the training phase. Common machine-learning algorithms are NNs, support vector machines, and fuzzy and recurrent negative correlation learners. Physical forecasting techniques predict the future states of variables based on mathematical models of physical processes and they include numerical weather prediction (NWP), engineering white and grey box, spatial averaging temporal interpolation and physical weather forecasting methods using external inputs. In these methods, the historical data are not generally needed and therefore avoid guessing the input-output relationship like NN. However, the parameterisation of the physical system sometimes has to be made by the accessibility of data sources 
that create complexity. As each method has its own weaknesses and strengths, hybrid ones, such as PSO-NN, NWP-NN and ARMA-NN, which take the strengths of each method, are applied in the literature [254, 255].

A large-scale spatial technique for providing very short-term probabilistic forecasting for different wind firms in Australia is demonstrated in [256, with a logit-normal transformation associated with a spatio-temporal model. The sVAR technique outperforms the conventional VAR and autoregressive models in terms of both the deterministic and probabilistic scores. A NWP and Gaussian process regression model considering the impact of atmospheric stability on the accuracy of short-term wind predictions, where the Gaussian model enhances the prediction accuracy over that of the original NWP data, are presented in [257]. A hybrid forecasting method consisting of a NN and metaheuristic algorithm, in which the latter is used to efficiently train the NN to avoid the trapping of local optima and over-fitting, is described in [258]. It is shown that this method obtains better prediction accuracy than nine others. In 259, a comparative study of forecasting methods for thirty-two solar plants of various sizes and technologies is presented. It also analyses the effects of the weather conditions and forecasts on the prediction of solar power generation. A hybrid method consisting of a generalised extreme learning machine, wavelet pre-processing and bootstrapping for improving the accuracy of load forecasting which considers the uncertainties of the forecasting model and noise data is discussed in [260]. In [261], four different deep-learning models for forecasting electricity prices are presented and it is concluded that they generally provide better accuracy than statistical models. However, there are various influential parameters in these estimating models in which improper parameter settings have an impact on the construction of these forecasting models in different ways, such as over- or under-fitting problems. For these reasons, parameters should be precisely chosen in which several optimisation techniques can be applied. There are still opportunities for researchers to construct a suitable forecasting model with higher accuracy, and minimum computational cost and complexity.

Energy management: Energy management involves the optimal use of energy for generated power and load demands taking into account some constraints, such as power, voltage, battery and logistics, in order to facilitate the economical, sustainable and reliable operation of a microgrid [262]. Due to the penetration of RESs, EVs and ESSs into existing networks, the general task of an energy management system has been diversified from the unit commitment and economical dispatch of generators. Therefore, the energy management of a microgrid can be performed from either the generation or load side, as shown in Figure 12. Although, conventionally, it involves the former, it is a challenging task due to the second-to-second power balancing required for stochastic power generation from RESs and fluctuating power demands. Energy management is the essential task in optimal scheduling for managing generation dispatch, saving energy, reducing system losses, supporting reactive power, mitigating GHG emissions, decreasing operational costs, and increasing reliability and efficiency of a microgrid [263. It also takes into account the operation, maintenance and degradation costs of a battery, and the penalty cost of the load shedding [117. In an energy management unit shown in Figure 9 firstly, a day-ahead scheduling based on the electricity price and forecasted data of power generation and demand is determined to obtain a cost-effective operation. Then, the short-term/real-time dispatch module receives the set-points from the scheduling program for sending them to the resources as commanded, compensating forecasted errors using short-term forecasting and real-time measurement of the data after each time step in the prediction horizon. Several computational algorithms, such as linear/non-linear programming, DP, GAs, fuzzy logic and NNs, are applied to reduce computational complexity and improve the convergence of energy 
management strategies for the efficient and reliable operation of a microgrid.

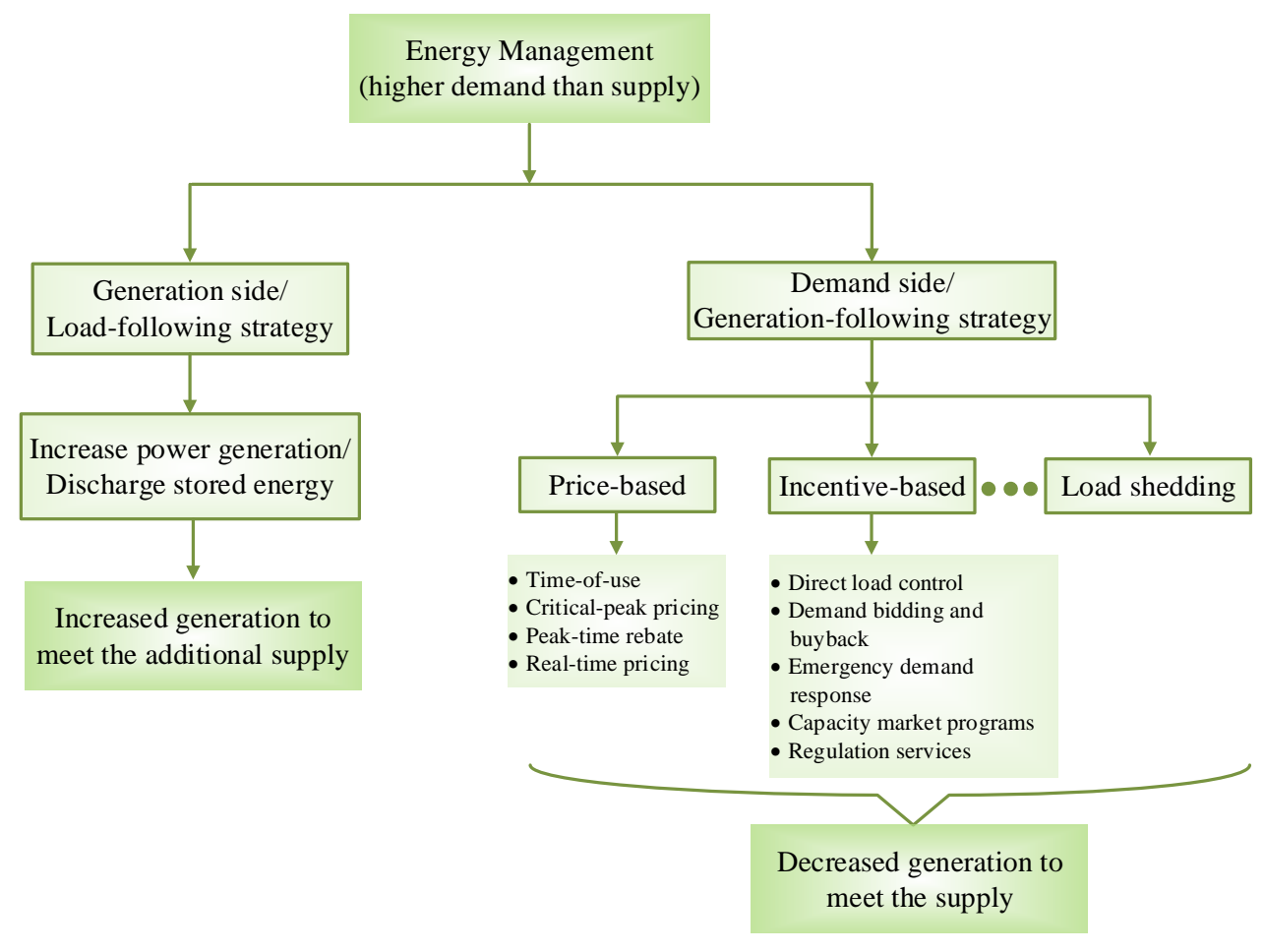

Figure 12: Energy management of microgrid including demand responses.

The linear programming model for minimising load consumption which balances the power supply and demand presented in 264 demonstrates that demand fluctuations can be reduced by up to $19 \%$. A costminimisation model for the optimal energy management of a grid-connected microgrid is described in [265. incorporation of different objective functions to find optimal operational points with reduced computational 
time and complexity.

Demand response: The increasing integration of RESs in a microgrid leads to difficulty in balancing its power supply and demand. Although this can be achieved using spinning reserves or ESSs, it incurs a significant investment cost. Therefore, energy operators emphasise a demand response that balances the power supply and demand based on customers' participation. This is because of its involvement in generation-following strategies that respond to the availability of power generation rather than load-following ones, as shown in Figure 12 As, traditionally, energy providers do not provide incentives for consumers to change their load patterns, clients are unaware of ways to efficiently use energy [273. However, those who exhibit a demand response by changing their power consumption in relation to the market's electricity prices are currently encouraged by the provision of different types of programs, such as direct load control, the emergency demand response program (EDRP), demand bidding, capacity market programs, interruptible rates, time-of-use (TOU), real-time pricing (RTP) and critical-peak pricing (CPP) 274, 275, 276, 277, 278, 279. These programs have their own unique features that determine their suitability for a particular community and basically offer financial incentives or impose penalties to encourage customers to use their loads flexibly. Some loads that a customer can reschedule according to the response of a program are those of air-conditioning systems, water heaters, EVs, washing machines and pumps. A demand response can help to achieve peak-load shaving, improve energy efficiency, decrease generation capacity, manage electricity costs, ensure network security, save energy and increase grid reliability. Also, it can maximise the capacity of a network by deferring the need for new investment in constructing the lines required by increased power demand [280, 281]. Load-shaping techniques using demand responses are shown in Figure 13 .

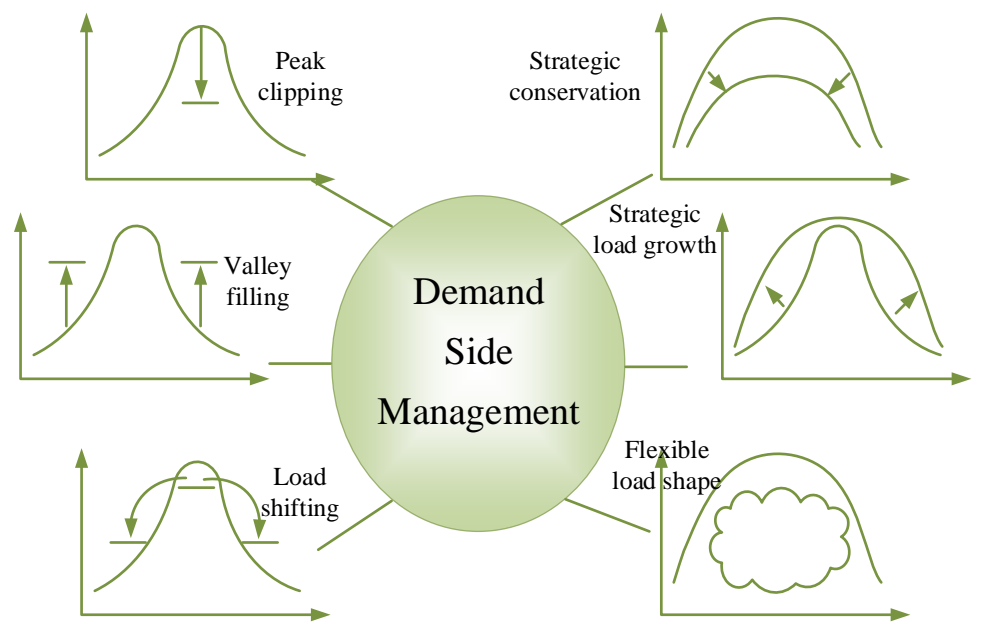

Figure 13: Demand-side management for load-shaping techniques [282].

Economic operations based on the demand responses of a microgrid are investigated in 283, 284, 285, and various price-based offers aimed at encouraging customers to participate in programs discussed in [283. In 284, interruptible load-based programs available for a variety of consumers are demonstrated while a real-time load-scheduling algorithm for the short-term power management of RESs at a minimum reserve cost is presented in 285]. An optimal economic dispatch of grid-connected microgrids considering the demand response which aims to minimise the effect of intermittent RESs and provide incentives to consumers is discussed in [277. In [278, the effects of irrational incentive values and consumers' participation in an EDRP in the presence 
of various uncertainties are analysed, and it is concluded that the optimum operational cost is related to the accurate selection of incentive values. A robust optimisation approach that considers an uncertainty model of upstream grid prices to obtain the optimal strategy for a microgrid to bid in a day-ahead market is proposed in 279 .

A financial-based demand response still suffers from promoting its rapid acceptance by electricity clients [286] while the demand response to a real-time price requires modifying the hardware on both the utility and consumer sides. As a result, both ageing utility workers and customers are unwilling to accept any quick changes in terms of technological infrastructures, e.g., the installation of communication lines and smart meters, which are perceived as intrusive [287. Therefore, educating the society about recent advance in technologies is essential to realise a large scale implementation of the demand response, otherwise it may take longer than expected to observe its widespread effectiveness.

In future, the demand response will be able to be realised in ways other than program-based ones as the number of prosumers (producer+consumers) is gradually increasing. Then, peer-to-peer (P2P) energy trading with the help of smart devices that facilitate each prosumer to take part in energy trading with one another will become an easy option for reducing electricity costs and energy storage losses, and optimising the output from RESs. Thus, the energy operators should take into account the rules and regulations about their power exchanging trades 288. In addition, as the energy trading deals with the decision-making process of participants with their own interests, the process needs to be modelled based on mathematical and signal processing tools to guarantee an efficient operation. The main challenges of the P2P energy trading is to model user behaviour, ensure the users' privacy, design pricing schemes and maintain trust between users 289. There are a lot of opportunity to improve the P2P energy trading in the future smart grid.

Coordination among microgrids: A distribution network can contain a large-scale integration of RESs through clusters of microgrids shown in Figure 7, called a multi-microgrid, that can be operated as a reliable power supply in the network during major external events. The formation of a multi-microgrid can enhance the economic and operational performance of each individual microgrid by reducing its energy exchange with the main grid. However, achieving the optimal operation of different microgrids is a challenging task, particularly under the influences of irregular RESs 290] because of the number of energy exchanges among the microgrids; for example, every microgrid firstly satisfies its internal power balance and then the amount of surplus power it supplies depends on the operations of the other microgrids in finding suitable energy trades within the multimicrogrid. Subsequently, a multi-microgrid is required to check whether it needs further energy exchanges with the grid to comply with the energy balance constraints. Also, each microgrid has its own profit-driven goals (local optima) that may contradict the objectives of the system operator (the global optimum).

The multi-microgrid concept was first introduced in 291 in which its technical feasibilities and complexities for providing primary reserves are investigated. In 292, a hierarchical control method for dealing with the frequency issues of a multi-microgrid is presented. A framework for operating microgrids at various frequencies, in which each microgrid is connected to a common dc line through an interlinking inverter controlled using a droop frequency to reduce the number of interlinking converters, is demonstrated in 293. It is also shown that the frequency control can be improved using the energy reserve of neighbouring microgrids which enable a high level of integration of RESs without the need for a large energy reserve. A coordinated management strategy for voltage and frequency controls that allows for a large number of RESs in a multi-microgrid and 
uses a meta-heuristic algorithm to support the voltage and a hierarchical structure to regulate the frequency during a transition is presented in 294 .

There are several publications in the literature that deal with the interactions in a multi-microgrid. A scheme for energy trading with scheduling using the Nash bargaining theory to encourage proactive energy trading and benefit sharing is developed in 295. A multi-objective approach for developing a fair scheme among microgrids, the power grid and the independent system operator to avoid conventional utility maximisation is presented in [296. An optimised strategy that considers DG units, storage systems, EVs and demand responses to assess the energy management of microgrids is described in [297. In [298, a distributed energy management technique for minimising a multi-microgrid's operating cost through exchanging power with neighbouring microgrids and the grid utility in a real-time market is developed. An analytical target-cascading theory for decoupling the dynamic economic dispatch between a multi-microgrid and grid utility to improve the power dispatch's efficiency is discussed in 299. In 290, a two-stage robust optimisation algorithm that considers discrete aggregates of the energy transactions among microgrids to reduce the operating cost is demonstrated. The first stage is used to buy/sell decisions and the second to optimise the power to be bought/sold under the worst-case energy scenarios. A study of cooperative game theory for achieving a highly energy-efficient operation of a multimicrogrid is presented in [300. It considers the potential conflicts of interest when maximising profits between microgrids' owners and system operators to obtain a trade-off solution, and concludes that power exchanges among microgrids can decrease the cost of power generation. A contingency-based energy management scheme for preventing a network from incurring economic loss is developed in [301]. It formulates the probability of contingencies in the energy management system for a multi-microgrid to avoid their effects in terms of economic losses. An energy trading using a game theory to balance the power generation and consumption of a multimicrogrid is designed in 302 and the basic structures of those networks from various sides can be found in 9. The work of a multi-microgrid can be further extended to enhance the energy efficiency and reliability by mathematically modelling different scenarios and uncertainties.

Smart meters and cyber attacks: Demand-side management can be facilitated by employing smart devices, such as smart meters and communication links, at consumers' premises to obtain the necessary data using advanced measurements and acting accordingly. Their communication links can assist in controlling the power demand by sending hourly electricity prices, which are usually high at times of peak load demands, from the utility to customers to shift flexible loads, such as washing machines, dishwashers and water heaters 303. As the electricity price depends on the power demands on a network, it is essential to access all the data on those of consumers, with an explicit-duration hidden Markov model with differential observations that receive these data from home appliances developed in [304. An energy management scheme with smart meters is demonstrated in 305 using a SCADA system that monitors a network of programmable logic controllers (PLCs) to collect various types of information from modern buildings.

There are some challenges related to implementing and operating an advanced metering infrastructure (AMI) due to the precision required for data reading and transmission, and its use of a high frequency that introduces privacy issues regarding consumers' data 306 because, as data are transmitted through WLAN/HAN/NAN networks, cyber-attacks by external controllers may steal information. A load-hiding approach that conceals the actual consumption of loads using storage systems is designed in [306. In [307, information leakage from a smart meter is studied using a RES and battery to partially hide customer details, and it is concluded that 
larger batteries can help to enhance privacy by exploiting the available energy. To determine malicious meters, a fraud detection system (FDS) is developed in [308] while detectors in both centralised and distributed settings for the online detection of false data injection (FDI) and denial of service (DoS) attacks are proposed in 309] using a CUSUM algorithm. As exposure to radio frequencies from smart meters during wireless communication is another concern, various countries have taken action to limit its bad effects [310]. A state-of-the-art survey of cyber security in 311 demonstrates its risks and offers solutions to the problem of increasing the security of power systems. New detection methods to protect information from cyber-attack need to be continuously developed with the advanced of technologies.

Regulatory policies: Regulatory policies are the principal motivation for customers and investor-owned electricity utilities to construct microgrids in order to obtain an effective and economical energy supply. The implementation of a microgrid can be facilitated through policies that prescribe guidelines for integrating DG units in it and connecting it to the grid utility. The current regulatory process is complex due to the amalgamation of different existing rules and regulations that are not particularly suitable for microgrids [312]. Although they are designed to maintain standard operations during a disturbance in a microgrid 313, the high connection fees are the main burden for microgrid operators 314. Also, the islanding operation, one of the most-cited features of microgrids, creates conflict with existing rules and regulations as it has a large effect on the power supply of a grid utility. Furthermore, bidirectional meters that need to be installed at the PCC to record energy exchanges between a microgrid and grid utility are another important issue. More research is still needed to realise the full benefits and thereby perceive politicians or lawmakers to amend the current laws in favour of constructing a microgrid.

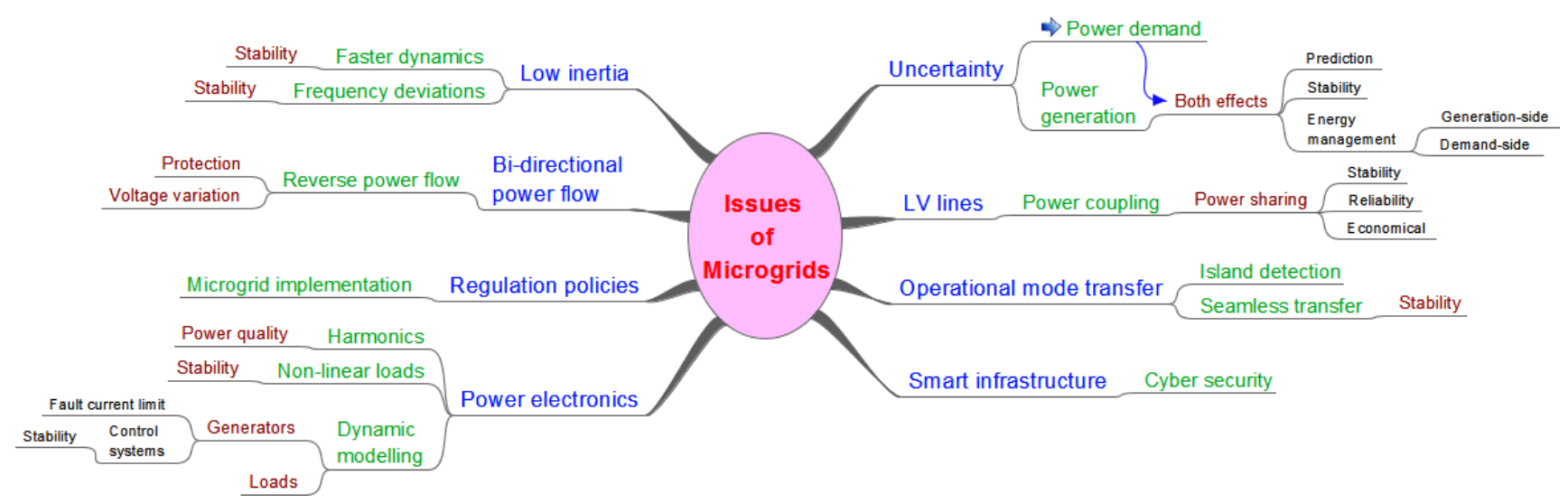

Figure 14: Pictorial elaboration of key issues for microgrids.

Remark 2: Numerous challenges that are barriers to fully exploiting the benefits of a microgrid can be identified from the above discussion. Of them, the key issues that lead to other difficulties are shown in Figure 14 and summarised as follows.

- Uncertainty: The stochastic nature of a RES, together with its non-dispatchable power generation, which leads to a generation-following strategy, is the greatest challenge for managing the second-to-second power balance of a microgrid for its economic, reliable and efficient operation. In addition, the uncertainty in power demand, which is much greater in a microgrid than bulk power system because of the limited number of load participants, is another challenge. 
- Low inertia: The dynamics of converter-interfaced DG units are almost neglected, except for those of the filters of an inverter and the capacitor at the dc-bus, compared with those of synchronous generators. Therefore, any change in a network prompts its DG units to respond quickly which may lead to instability because of the severe frequency deviations of an islanded microgrid.

- LV distribution lines: The LV lines, in which RESs are integrated, are dominated mainly by resistance rather than inductance. Therefore, conventional control theories need to be revised as there is a strong coupling in both the active and reactive powers which leads to power-sharing issues. prices and a microgrid operator can manage the demand responses.

Economic benefits: If a microgrid is constructed on an island for geographical reasons, the community can benefit by operating small-scale factories and irrigation to improve its lifestyle and economy. Also, business 
activity is increased due to access to electricity for freezing and heating systems, and other modern amenities. Moreover, the RESs used in a microgrid are environmentally friendly and can provide an island with sustainable development.

Improved stability of grid network: Microgrids are self-controlled entities from the grid utility perspective due to their own operating capabilities during any undesirable situation, such as a major storm or fault on the main grid. These features enable them to simultaneously improve their stability and that of the grid utility because they can detect any malfunction within their regions and act accordingly to protect themselves and the grid utility against cascaded failures.

High integration of RESs: RESs are stochastic in nature due to their high dependence on the weather; for example, solar energy is produced from solar irradiation and wind power from the wind's speed. Although integrating them in a distribution network poses challenges, these can be overcome by using a central control approach for a microgrid. Most importantly, a high penetration of RESs in a microgrid reduces carbon emissions, thereby providing socio-economic and environmental benefits.

\section{Future work}

As a microgrid has evolved from a distribution network and can be viewed as a building block of a smart grid supported by modern communication architectures, microgrid research is likely to continue until smart grids are fully established everywhere. Although there are different projects on converter-based microgrids, most are implemented as test beds and there are still several challenges that need to be addressed before realising the full potential of microgrids. Different approaches developed with the aim of applying a particular solution method have some limitations. As extensive research on microgrids has been conducted during the last decade, some issues, such as their inner control and power sharing, have been resolved to some extent. However, as it is still possible to develop better techniques for improving a microgrid's performance, future work is likely to be conducted in the following areas.

- Advanced control techniques (such as artificial intelligence, predictive control and MASs) considering high penetrations of RESs with different power ratings need to be designed/implemented to resolve issues regarding power quality and power sharing. However, proper power sharing for RESs, which is in a practical field, is often overlooked as it is assumed that the voltage source at the inputs to a converter is ideal.

- As an effective control application depends on the accuracy of the model of a system, the uncertainties in a microgrid, for example, those of catastrophes, power and loads, should also be modelled when designing an advanced controller, the complexity of which can be reduced for implementation in practical systems.

- The low inertia of DG units may experience severe voltage and/or frequency changes during abrupt disturbances. Although there is some previous research that increases the response times of DG units by applying a flywheel and/or mimicking synchronous generators, there are still opportunities for researchers to develop a microgrid with improved inertia.

- Maintaining the stable operation of a microgrid is challenging due to the increased participation of nonlinear loads and high penetration of DG units. Although the stabilities of microgrids with linear loads in a radial network have been extensively studied recently, determining the stability margins of a mesh network for DG units and synchronous generators with non-linear loads, such as induction motors, constant power loads 
and electrical vehicles, has not been thoroughly investigated.

- Power management of the generation side of a conventional power system using several optimisation a modern distribution network, a high penetration of RESs is restricted due to severe issues regarding the grid utility whereas a microgrid is capable of overcoming these problems because of its unique regulation features, such as having a specific area controlled by an MGCC. Although the microgrid concept was introduced back in 2002, there is a no clear distinction between a microgrid and a distribution network due to their very similar

\section{Acknowledgment}

This work is supported by the Australian Government Research Training Program (RTP) Scholarship at The University of New South Wales - Canberra, Australia. Md. Alamgir Hossain is thankful to Naruttam Kumar Roy, Shafiul Alam, Utpal Kumar Das and Adnan Anwar for their valuable comments. 


\section{References}

[17] C. Breidenich, D. Magraw, A. Rowley, J. W. Rubin, The kyoto protocol to the united nations framework convention on climate change, The American Journal of International Law 92 (2) (1998) 315-331.

1] M. A. Hossain, H. Pota, M. J. Hossain, A. M. O. Haruni, Active power management in a low-voltage islanded microgrid, International Journal of Electrical Power \& Energy Systems 98 (2018) 36-48.

[2] M. A. Hossain, M. R. Ahmed, Present energy scenario and potentiality of wind energy in bangladesh, World Academy of Science, Engineering and Technology 7 (11) (2013) 1001-1005.

[3] E. Martinot, et al., Renewables 2018: Global status report, Worldwatch Institute Washington, DC, 2018.

[4] M. A. Hossain, H. R. Pota, W. Issa, M. J. Hossain, Overview of ac microgrid controls with inverterinterfaced generations, Energies 10 (9) (2017) 1300.

[5] J. S. M. Ali, V. Krishnaswamy, An assessment of recent multilevel inverter topologies with reduced power electronics components for renewable applications, Renewable and Sustainable Energy Reviews 82 (2018) 3379-3399.

[6] M. S. Mahmoud, N. M. Alyazidi, M. I. Abouheaf, Adaptive intelligent techniques for microgrid control systems: A survey, International Journal of Electrical Power \& Energy Systems 90 (2017) 292-305.

[7] Z. Shuai, Y. Sun, Z. J. Shen, W. Tian, C. Tu, Y. Li, X. Yin, Microgrid stability: Classification and a review, Renewable and Sustainable Energy Reviews 58 (2016) 167-179.

[8] M. W. Khan, J. Wang, The research on multi-agent system for microgrid control and optimization, Renewable and Sustainable Energy Reviews 80 (2017) 1399-1411.

[9] Z. Xu, P. Yang, C. Zheng, Y. Zhang, J. Peng, Z. Zeng, Analysis on the organization and development of multi-microgrids, Renewable and Sustainable energy reviews 81 (2018) 2204-2216.

[10] F. Martin-Martínez, A. Sánchez-Miralles, M. Rivier, A literature review of microgrids: A functional layer based classification, Renewable and Sustainable Energy Reviews 62 (2016) 1133-1153.

[11] K. Milis, H. Peremans, S. Van Passel, The impact of policy on microgrid economics: A review, Renewable and Sustainable Energy Reviews 81 (2018) 3111-3119.

[12] E. Planas, J. Andreu, J. I. Gárate, I. M. de Alegría, E. Ibarra, Ac and dc technology in microgrids: A review, Renewable and Sustainable Energy Reviews 43 (2015) 726-749.

[13] M. Hossain, H. Pota, A. Haruni, M. Hossain, Dc-link voltage regulation of inverters to enhance microgrid stability during network contingencies, Electric Power Systems Research 147 (2017) 233-244.

[14] S. Kakran, S. Chanana, Smart operations of smart grids integrated with distributed generation: A review, Renewable and Sustainable Energy Reviews 81 (2018) 524-535.

[15] N. Jenkins, A. Ron, C. Peter, K. Daniel, S. Goran, Embedded generation, no. 31, IET, 2000.

[16] T. A. Short, Electric power distribution handbook, CRC press, 2014. 
[18] D. G. CIRED, Preliminary report of CIRED (international conference on electricity distribution), Working Group WG04, Brussels, Belgium (1999).

[19] B. Singh, V. Mukherjee, P. Tiwari, A survey on impact assessment of DG and FACTS controllers in power systems, Renewable and Sustainable Energy Reviews 42 (2015) 846-882.

[20] I. B. Bjelic, R. M. Ciric, Optimal distributed generation planning at a local level-a review of serbian renewable energy development, Renewable and Sustainable Energy Reviews 39 (2014) 79-86.

[21] O. M. Longe, K. Ouahada, S. Rimer, H. C. Ferreira, A. Han Vinck, Distributed optimisation algorithm for demand side management in a grid-connected smart microgrid, Sustainability 9 (7) (2017) 1088.

[22] R. Adib, H. Murdock, F. Appavou, A. Brown, B. Epp, A. Leidreiter, C. Lins, H. Murdock, E. Musolino, K. Petrichenko, et al., Renewables 2016 global status report (2015).

[23] S. Barsali, M. Ceraolo, P. Pelacchi, D. Poli, Control techniques of dispersed generators to improve the continuity of electricity supply, in: Power Engineering Society Winter Meeting, 2002. IEEE, Vol. 2, IEEE, 2002, pp. 789-794.

[24] N. Bottrell, Small-signal analysis of active loads and large-signal analysis of faults in inverter interfaced microgrid applications, Doctoral dissertation (2013), Imperial College London.

[25] P. Chiradeja, Benefit of distributed generation: a line loss reduction analysis, in: Transmission and Distribution Conference and Exhibition: Asia and Pacific, 2005 IEEE/PES, IEEE, 2005, pp. 1-5.

[26] T. E. McDermott, R. C. Dugan, Distributed generation impact on reliability and power quality indices, in: Rural Electric Power Conference, 2002 IEEE, IEEE, 2002, pp. D3-1.

[27] G. Shafiullah, A. M. Oo, D. Jarvis, A. S. Ali, P. Wolfs, Potential challenges: Integrating renewable energy with the smart grid, in: Universities Power Engineering Conference (AUPEC), 2010 20th Australasian, IEEE, 2010, pp. 1-6.

[28] A. Piccolo, P. Siano, Evaluating the impact of network investment deferral on distributed generation expansion, Power Systems, IEEE Transactions on 24 (3) (2009) 1559-1567.

[29] B. Singh, A. Chandra, K. Al-Haddad, Power quality: problems and mitigation techniques, John Wiley \& Sons, 2014.

[30] H. B. Puettgen, P. R. Macgregor, F. C. Lambert, Distributed generation: Semantic hype or the dawn of a new era?, Power and Energy Magazine, IEEE 1 (1) (2003) 22-29.

[31] Y. Li, D. M. Vilathgamuwa, P. C. Loh, Microgrid power quality enhancement using a three-phase four-wire grid-interfacing compensator, Industry Applications, IEEE Transactions on 41 (6) (2005) 1707-1719.

[32] S. Engelhardt, I. Erlich, C. Feltes, J. Kretschmann, F. Shewarega, Reactive power capability of wind turbines based on doubly fed induction generators, Energy Conversion, IEEE Transactions on 26 (1) (2011) 364-372. 
[45] H. F. Habib, A. Mohamed, M. El Hariri, O. A. Mohammed, Utilizing supercapacitors for resiliency enhancements and adaptive microgrid protection against communication failures, Electric Power Systems Research 145 (2017) 223-233.

[33] R. Teodorescu, M. Liserre, P. Rodriguez, Grid converters for photovoltaic and wind power systems, Vol. 29, John Wiley \& Sons, 2011.

[34] R. Kumar, B. Singh, D. Shahani, C. Jain, Dual-tree complex wavelet transform-based control algorithm for power quality improvement in a distribution system, IEEE Transactions on Industrial Electronics 64 (1) (2017) 764-772.

[35] R. Kalpana, B. Singh, G. Bhuvaneswari, et al., A 20-pulse asymmetric multiphase staggering autoconfigured transformer for power quality improvement, IEEE Transactions on Power Electronics 33 (2) (2018) $917-925$.

[36] P. P. Barker, R. W. De Mello, Determining the impact of distributed generation on power systems. I. Radial distribution systems, in: Power Engineering Society Summer Meeting, 2000. IEEE, Vol. 3, IEEE, 2000, pp. 1645-1656.

[37] M. Moghbel, M. A. Masoum, A. Fereidouni, S. Deilami, Optimal sizing, siting and operation of custom power devices with statcom and aplc functions for real-time reactive power and network voltage quality control of smart grid, IEEE Transactions on Smart Grid 9 (2017) 5564 - 5575.

[38] P. Prabhakar, A. Kumar, Voltage stability boundary and margin enhancement with FACTS and HVDC, International Journal of Electrical Power \& Energy Systems 82 (2016) 429-438.

[39] R. Sirjani, A. R. Jordehi, Optimal placement and sizing of distribution static compensator (D-STATCOM) in electric distribution networks: A review, Renewable and Sustainable Energy Reviews 77 (2017) 688694.

[40] X. Ge, F. Gao, Flexible third harmonic voltage control of low capacitance cascaded h-bridge statcom, IEEE Transactions on Power Electronics 33 (3) (2018) 1884-1889.

[41] P. M. Sotkiewicz, J. M. Vignolo, Nodal pricing for distribution networks: efficient pricing for efficiency enhancing DG, Power Systems, IEEE Transactions on 21 (2) (2006) 1013.

[42] P. Srithorn, Control of a STATCOM with supercapacitor energy storage, Ph.D. thesis, University of Nottingham (2009).

[43] M. Kavi, Y. Mishra, M. Vilathgamuwa, Morphological fault detector for adaptive overcurrent protection in distribution networks with increasing photovoltaic penetration, IEEE Transactions on Sustainable Energy 9 (3) (2018) 1021-1029.

[44] V. A. Papaspiliotopoulos, G. N. Korres, V. A. Kleftakis, N. D. Hatziargyriou, Hardware-in-the-loop design and optimal setting of adaptive protection schemes for distribution systems with distributed generation, IEEE Transactions on Power Delivery 32 (1) (2017) 393-400. 
[46] H. R. Baghaee, M. Mirsalim, G. B. Gharehpetian, H. A. Talebi, A new current limiting strategy and fault model to improve fault ride-through capability of inverter interfaced ders in autonomous microgrids, Sustainable Energy Technologies and Assessments 24 (2017) 71-81.

[47] S. B. Naderi, M. Negnevitsky, A. Jalilian, M. T. Hagh, Efficient fault ride-through scheme for three phase voltage source inverter-interfaced distributed generation using dc link adjustable resistive type fault current limiter, Renewable Energy 92 (2016) 484-498.

[48] M. Shafiul Alam, M. A. Y. Abido, Fault ride-through capability enhancement of voltage source converterhigh voltage direct current systems with bridge type fault current limiters, Energies 10 (11) (2017) 1898.

[49] I. Xyngi, A. Ishchenko, M. Popov, L. van der Sluis, Transient stability analysis of a distribution network with distributed generators, IEEE Transactions on Power Systems 24 (2) (2009) 1102-1104.

[50] C. Wang, K. Yuan, P. Li, B. Jiao, G. Song, A projective integration method for transient stability assessment of power systems with a high penetration of distributed generation, IEEE Transactions on Smart Grid 9 (1) (2018) 386-395.

[51] M. Benidris, J. Mitra, C. Singh, Integrated evaluation of reliability and stability of power systems, IEEE Transactions on Power Systems 32 (5) (2017) 4131-4139.

[52] Y. Xu, Z. Yang, W. Gu, M. Li, Z. Deng, Robust real-time distributed optimal control based energy management in a smart grid, IEEE Transactions on Smart Grid 8 (4) (2017) 1568-1579.

[53] M. Bakhshi, M. H. Holakooie, A. Rabiee, Fuzzy based damping controller for TCSC using local measurements to enhance transient stability of power systems, International Journal of Electrical Power \& Energy Systems 85 (2017) 12-21.

[54] Z. A. Obaid, L. Cipcigan, M. T. Muhssin, Power system oscillations and control: Classifications and psss design methods: A review, Renewable and Sustainable Energy Reviews 79 (2017) 839-849.

[55] P. Kundur, J. Paserba, V. Ajjarapu, G. Andersson, A. Bose, C. Canizares, N. Hatziargyriou, D. Hill, A. Stankovic, C. Taylor, et al., Definition and classification of power system stability IEEE/CIGRE joint task force on stability terms and definitions, IEEE transactions on Power Systems 19 (3) (2004) 1387-1401.

[56] M. D. Ilic, J. Zaborszky, Dynamics and control of large electric power systems, Wiley New York, 2000.

[57] Y. Levron, J. Belikov, Modeling power networks using dynamic phasors in the dq0 reference frame, Electric Power Systems Research 144 (2017) 233-242.

[58] S. Almer, U. Jonsson, Dynamic phasor analysis of periodic systems, IEEE Transactions on automatic control 54 (8) (2009) 2007-2012.

[59] M. Daryabak, S. Filizadeh, J. Jatskevich, A. Davoudi, M. Saeedifard, V. Sood, J. Martinez, D. Aliprantis, J. Cano, A. Mehrizi-Sani, Modeling of LCC-HVDC systems using dynamic phasors, IEEE Transactions on Power Delivery 29 (4) (2014) 1989-1998. 
[60] R. Yousefian, S. Kamalasadan, A lyapunov function based optimal hybrid power system controller for improved transient stability, Electric Power Systems Research 137 (2016) 6-15.

[71] A. Attya, S. Ademi, M. Jovanović, O. Anaya-Lara, Frequency support using doubly fed induction and reluctance wind turbine generators, International Journal of Electrical Power \& Energy Systems 101 (2018) 403-414.

[72] H. Knudsen, J. N. Nielsen, Introduction to the modeling of wind turbines, Wind power in power systems

[73] M. A. Hossain, H. R. Pota, S. Squartini, F. Zaman, K. M. Muttaqi, Energy management of community microgrids considering degradation cost of battery, Journal of Energy Storage, 2019.

[74] I. Serban, C. Marinescu, Control strategy of three-phase battery energy storage systems for frequency support in microgrids and with uninterrupted supply of local loads, IEEE Transactions on Power Electronics 
[75] Q.-C. Zhong, G. Weiss, Synchronverters: Inverters that mimic synchronous generators, IEEE Transactions on Industrial Electronics 58 (4) (2011) 1259-1267.

[76] Q.-C. Zhong, G. C. Konstantopoulos, B. Ren, M. Krstic, Improved synchronverters with bounded frequency and voltage for smart grid integration, IEEE Transactions on Smart Grid 9 (2) (2018) 786-796.

[89] F. Katiraei, R. Iravani, N. Hatziargyriou, A. Dimeas, Microgrids management, IEEE Power Energy Magagine 6 (3) (2008) 54-65.

[90] K. Rajesh, S. Dash, R. Rajagopal, R. Sridhar, A review on control of ac microgrid, Renewable and Sustainable Energy Reviews 71 (2017) 814-819. 
[102] J. Rocabert, A. Luna, F. Blaabjerg, P. Rodrguez, Control of power converters in ac microgrids, Power Electronics, IEEE Transactions on 27 (11) (2012) 4734-4749.

[103] J. G. de Matos, F. SF e Silva, R. de S, A. Luiz, Power control in ac isolated microgrids with renewable energy sources and energy storage systems, Industrial Electronics, IEEE Transactions on 62 (6) (2015)

[104] D. Nilsson, A. Sannino, Efficiency analysis of low-and medium-voltage dc distribution systems, in: Power Engineering Society General Meeting, 2004. IEEE, IEEE, 2004, pp. 2315-2321. 
[105] J. G. Ciezki, R. W. Ashton, Selection and stability issues associated with a navy shipboard dc zonal electric distribution system, Power Delivery, IEEE Transactions on 15 (2) (2000) 665-669.

[106] A. T. Elsayed, A. A. Mohamed, O. A. Mohammed, DC microgrids and distribution systems: An overview, Electric Power Systems Research 119 (2015) 407-417.

[107] D. Salomonsson, A. Sannino, Low-voltage dc distribution system for commercial power systems with sensitive electronic loads, Power Delivery, IEEE Transactions on 22 (3) (2007) 1620-1627.

[108] F. Nejabatkhah, Y. W. Li, Overview of power management strategies of hybrid ac/dc microgrid, Power Electronics, IEEE Transactions on 30 (12) (2015) 7072-7089.

[109] H. Xiao, A. Luo, Z. Shuai, G. Jin, Y. Huang, An improved control method for multiple bidirectional power converters in hybrid ac/dc microgrid, Smart Grid, IEEE Transactions on 7 (1) (2016) 340-347.

[110] L. Che, M. Shahidehpour, A. Alabdulwahab, Y. Al-Turki, Hierarchical coordination of a community microgrid with ac and dc microgrids, Smart Grid, IEEE Transactions on 6 (6) (2015) 3042-3051.

[111] Q. Jiang, M. Xue, G. Geng, Energy management of microgrid in grid-connected and stand-alone modes, IEEE transactions on power systems 28 (3) (2013) 3380-3389.

[112] A. Vinayagam, A. A. Alqumsan, K. Swarna, S. Y. Khoo, A. Stojcevski, Intelligent control strategy in the islanded network of a solar PV microgrid, Electric Power Systems Research 155 (2018) 93-103.

[113] M. A. Hossain, H. R. Pota, Voltage tracking of a single-phase inverter in an islanded microgrid, International Journal of Renewable Energy Research (IJRER) 5 (3) (2015) 806-814.

[114] Y. Yang, K. Zhou, F. Blaabjerg, Current harmonics from single-phase grid-connected invertersexamination and suppression, Emerging and Selected Topics in Power Electronics, IEEE Journal of 4 (3) (2016) 221-233.

[115] Q.-C. Zhong, T. Hornik, Cascaded current-voltage control to improve the power quality for a gridconnected inverter with a local load, Industrial Electronics, IEEE Transactions on 60 (4) (2013) 13441355.

[116] I. E. Commission, et al., IEC 61970-301: Energy management system application program interface (EMSAPI)-part 301: Common information model (CIM) base, International Electrotechnical Commission $(2003)$.

[117] M. F. Zia, E. Elbouchikhi, M. Benbouzid, Microgrids energy management systems: A critical review on methods, solutions, and prospects, Applied Energy 222 (2018) 1033-1055.

[118] A. Bidram, A. Davoudi, Hierarchical structure of microgrids control system, IEEE Transactions on Smart Grid 3 (4) (2012) 1963-1976.

[119] J. M. Guerrero, J. C. Vasquez, J. Matas, L. G. De Vicuña, M. Castilla, Hierarchical control of droopcontrolled ac and de microgrids: A general approach toward standardization, IEEE Transactions on industrial electronics 58 (1) (2011) 158-172. 
[120] M. Rahman, A. Oo, Distributed multi-agent based coordinated power management and control strategy for microgrids with distributed energy resources, Energy Conversion and Management 139 (2017) 20-32.

[121] T. Vandoorn, J. Guerrero, J. De Kooning, J. Vásquez, L. Vandevelde, Decentralized and centralized control of islanded microgrids including reserve management, IEEE Ind Electron Mag (2013) 1-14.

[122] A. Anvari-Moghaddam, A. Rahimi-Kian, M. S. Mirian, J. M. Guerrero, A multi-agent based energy management solution for integrated buildings and microgrid system, Applied Energy 203 (2017) 41-56.

[123] M. A. Hossain, DC-link voltage control for reliable operation of low-voltage islanded microgrids, Master's thesis, The University of New South Wales, Australia (2016).

[124] J. Almada, R. Leão, R. Sampaio, G. Barroso, A centralized and heuristic approach for energy management of an ac microgrid, Renewable and Sustainable Energy Reviews 60 (2016) 1396-1404.

[125] L. Olatomiwa, S. Mekhilef, M. Ismail, M. Moghavvemi, Energy management strategies in hybrid renewable energy systems: A review, Renewable and Sustainable Energy Reviews 62 (2016) 821-835.

[126] C. Cho, J.-H. Jeon, J.-Y. Kim, S. Kwon, K. Park, S. Kim, Active synchronizing control of a microgrid, IEEE Transactions on Power Electronics 26 (12) (2011) 3707-3719.

[127] A. G. Tsikalakis, N. D. Hatziargyriou, Centralized control for optimizing microgrids operation, in: 2011 IEEE power and energy society general meeting, IEEE, 2011, pp. 1-8.

[128] A. A. Zaidi, F. Kupzog, Microgrid automation-a self-configuring approach, in: Multitopic Conference, 2008. INMIC 2008. IEEE International, IEEE, 2008, pp. 565-570.

[129] N. L. Diaz, A. C. Luna, J. C. Vasquez, J. M. Guerrero, Centralized control architecture for coordination of distributed renewable generation and energy storage in islanded ac microgrids, IEEE Transactions on Power Electronics pp (99) (2016) 1-1.

[130] J. M. Guerrero, P. C. Loh, T.-L. Lee, M. Chandorkar, Advanced control architectures for intelligent microgrids?part ii: Power quality, energy storage, and ac/dc microgrids, IEEE Transactions on Industrial Electronics 60 (4) (2013) 1263-1270.

[131] F. Shahnia, A. Arefi, Eigenanalysis-based small signal stability of the system of coupled sustainable microgrids, International Journal of Electrical Power \& Energy Systems 91 (2017) 42-60.

[132] L. Xu, Y. Wang, Dynamic modeling and control of DFIG-based wind turbines under unbalanced network conditions, IEEE Transactions on Power Systems 22 (1) (2007) 314-323.

[133] S. V. Iyer, M. N. Belur, M. C. Chandorkar, A generalized computational method to determine stability of a multi-inverter microgrid, IEEE Transactions on Power Electronics 25 (9) (2010) 2420-2432.

[134] B. Wen, D. Boroyevich, R. Burgos, P. Mattavelli, Z. Shen, Inverse nyquist stability criterion for grid-tied inverters, IEEE Transactions on Power Electronics 32 (2) (2017) 1548-1556. 
[135] B. Wen, D. Boroyevich, R. Burgos, P. Mattavelli, Z. Shen, Small-signal stability analysis of three-phase ac systems in the presence of constant power loads based on measured dq frame impedances, IEEE Transactions on Power Electronics 30 (10) (2015) 5952-5963.

[136] E. Planas, A. Gil-de Muro, J. Andreu, I. Kortabarria, I. M. de Alegría, Design and implementation of a droop control in $\mathrm{d}-\mathrm{q}$ frame for islanded microgrids, IET Renewable Power Generation 7 (5) (2013) 458-474.

[137] E. A. Coelho, P. C. Cortizo, P. F. D. Garcia, Small signal stability for single phase inverter connected to stiff ac system, in: Industry Applications Conference, 1999. Thirty-Fourth IAS Annual Meeting. Conference Record of the 1999 IEEE, Vol. 4, IEEE, 1999, pp. 2180-2187.

[138] I. P. NIKOLAKAKOS, H. H. Zeineldin, M. S. El Moursi, J. L. Kirtley, Reduced-order model for interinverter oscillations in islanded droop-controlled microgrids, IEEE Transactions on Smart Grid 9 (5) (2018) 4953-4963.

[139] M. H. Fini, M. E. H. Golshan, Determining optimal virtual inertia and frequency control parameters to preserve the frequency stability in islanded microgrids with high penetration of renewables, Electric Power Systems Research 154 (2018) 13-22.

[140] A. Luna, F. d. A. Lima, D. Santos, P. Rodríguez, E. H. Watanabe, S. Arnaltes, Simplified modeling of a DFIG for transient studies in wind power applications, IEEE Transactions on Industrial Electronics 58 (1) (2011) 9-20.

[141] S.-K. Kim, J.-H. Jeon, C.-H. Cho, E.-S. Kim, J.-B. Ahn, Modeling and simulation of a grid-connected PV generation system for electromagnetic transient analysis, Solar Energy 83 (5) (2009) 664-678.

[142] B. Tamimi, C. Cañizares, K. Bhattacharya, System stability impact of large-scale and distributed solar photovoltaic generation: The case of ontario, canada, IEEE transactions on sustainable energy 4 (3) (2013) 680-688.

[143] A. D. Paquette, D. M. Divan, Virtual impedance current limiting for inverters in microgrids with synchronous generators, IEEE Transactions on Industry Applications 51 (2) (2015) 1630-1638.

[144] H. Xin, L. Huang, L. Zhang, Z. Wang, J. Hu, Synchronous instability mechanism of pf droop-controlled voltage source converter caused by current saturation, IEEE Transactions on Power Systems 31 (6) (2016) 5206-5207.

[145] L. Huang, H. Xin, Z. Wang, L. Zhang, K. Wu, J. Hu, Transient stability analysis and control design of droop-controlled voltage source converters considering current limitation, IEEE Transactions on Smart Grid (2017) 1-1.

[146] M. Kabalan, P. Singh, D. Niebur, Nonlinear lyapunov stability analysis of seven models of a dc/ac droop controlled inverter connected to an infinite bus, IEEE Transactions on Smart Grid (2017).

[147] R. Majumder, Some aspects of stability in microgrids, IEEE Transactions on power systems 28 (3) (2013) 3243-3252. 
[148] J. M. Guerrero, L. G. De Vicuna, J. Matas, M. Castilla, J. Miret, A wireless controller to enhance dynamic performance of parallel inverters in distributed generation systems, IEEE Transactions on power electronics 19 (5) (2004) 1205-1213.

[149] R. Majumder, B. Chaudhuri, A. Ghosh, R. Majumder, G. Ledwich, F. Zare, Improvement of stability and load sharing in an autonomous microgrid using supplementary droop control loop, Power Systems, IEEE Transactions on 25 (2) (2010) 796-808.

[150] C. N. Rowe, T. J. Summers, R. E. Betz, D. J. Cornforth, T. G. Moore, Arctan power-frequency droop for improved microgrid stability, IEEE Transactions on Power Electronics 28 (8) (2013) 3747-3759.

[151] M. N. Ambia, A. Al-Durra, C. Caruana, S. M. Muyeen, Stability enhancement of a hybrid micro-grid system in grid fault condition, in: Electrical Machines and Systems (ICEMS), 2012 15th International Conference on, IEEE, 2012, pp. 1-6.

[152] J. Shiles, E. Wong, S. Rao, C. Sanden, M. Zamani, M. Davari, F. Katiraei, Microgrid protection: An overview of protection strategies in north american microgrid projects, in: Power \& Energy Society General Meeting, 2017 IEEE, IEEE, 2017, pp. 1-5.

[153] L. Che, M. E. Khodayar, M. Shahidehpour, Adaptive protection system for microgrids: Protection practices of a functional microgrid system., IEEE Electrification magazine 2 (1) (2014) 66-80.

[154] M. Monadi, M. A. Zamani, J. I. Candela, A. Luna, P. Rodriguez, Protection of ac and dc distribution systems embedding distributed energy resources: A comparative review and analysis, Renewable and sustainable energy reviews 51 (2015) 1578-1593.

[155] S. M. Brahma, A. A. Girgis, Microprocessor-based reclosing to coordinate fuse and recloser in a system with high penetration of distributed generation, in: Power Engineering Society Winter Meeting, 2002. IEEE, Vol. 1, IEEE, 2002, pp. 453-458.

[156] H. H. Zeineldin, Y. A.-R. I. Mohamed, V. Khadkikar, V. R. Pandi, A protection coordination index for evaluating distributed generation impacts on protection for meshed distribution systems, IEEE Transactions on Smart Grid 4 (3) (2013) 1523-1532.

[157] N. Hadjsaid, J.-F. Canard, F. Dumas, Dispersed generation impact on distribution networks, IEEE Computer Applications in power 12 (2) (1999) 22-28.

[158] H. A. Abdel-Ghany, A. M. Azmy, N. I. Elkalashy, E. M. Rashad, Optimizing dg penetration in distribution networks concerning protection schemes and technical impact, Electric Power Systems Research 128 (2015) $113-122$

[159] S. A. Hosseini, H. A. Abyaneh, S. H. H. Sadeghi, F. Razavi, A. Nasiri, An overview of microgrid protection methods and the factors involved, Renewable and Sustainable Energy Reviews 64 (2016) 174-186.

[160] W. K. Najy, H. H. Zeineldin, W. L. Woon, Optimal protection coordination for microgrids with gridconnected and islanded capability, IEEE Transactions on industrial electronics 60 (4) (2013) 1668-1677. 
[161] Ł. Huchel, H. H. Zeineldin, Planning the coordination of directional overcurrent relays for distribution systems considering dg, IEEE Transactions on Smart Grid 7 (3) (2016) 1642-1649.

[162] R. Chabanloo, H. A. Abyaneh, A. Agheli, H. Rastegar, Overcurrent relays coordination considering transient behaviour of fault current limiter and distributed generation in distribution power network, IET generation, transmission \& distribution 5 (9) (2011) 903-911.

[163] A. Y. Abdelaziz, H. Talaat, A. Nosseir, A. A. Hajjar, An adaptive protection scheme for optimal coordination of overcurrent relays, Electric Power Systems Research 61 (1) (2002) 1-9.

[164] M. N. Alam, Adaptive protection coordination scheme using numerical directional overcurrent relays, IEEE Transactions on Industrial Informatics (2018) 1-1.

[165] A. Oudalov, A. Fidigatti, Adaptive network protection in microgrids, International Journal of Distributed Energy Resources 5 (3) (2009) 201-226.

[166] S. Conti, Analysis of distribution network protection issues in presence of dispersed generation, Electric Power Systems Research 79 (1) (2009) 49-56.

[167] F. Ren, M. Zhang, D. Soetanto, X. Su, Conceptual design of a multi-agent system for interconnected power systems restoration, IEEE transactions on power systems 27 (2) (2012) 732-740.

[168] H. F. Habib, C. R. Lashway, O. A. Mohammed, A review of communication failure impacts on adaptive microgrid protection schemes and the use of energy storage as a contingency, IEEE Transactions on Industry Applications 54 (2) (2018) 1194-1207.

[169] J. Merino, P. Mendoza-Araya, G. Venkataramanan, M. Baysal, Islanding detection in microgrids using harmonic signatures, IEEE Transactions on Power Delivery 30 (5) (2015) 2102-2109. doi:10.1109/ TPWRD.2014.2383412.

[170] S. Dutta, P. K. Sadhu, M. J. B. Reddy, D. K. Mohanta, Shifting of research trends in islanding detection method-a comprehensive survey, Protection and Control of Modern Power Systems 3 (1) (2018) 1.

[171] B. M. Biaz, V. H. Ferreira, M. Z. Fortes, T. T. Lopes, G. B. A. Lima, Islanding detection in distributed generation using unsupervised learning techniques, IEEE Latin America Transactions 16 (1) (2018) 118125 .

[172] M. Mishra, M. Sahani, P. Rout, An islanding detection algorithm for distributed generation based on hilbert-huang transform and extreme learning machine, Sustainable Energy, Grids and Networks 9 (2017) $13-26$.

[173] H. Zeineldin, T. Abdel-Galil, E. El-Saadany, M. Salama, Islanding detection of grid connected distributed generators using TLS-ESPRIT, Electric Power Systems Research 77 (2) (2007) 155-162.

[174] T. S. Basso, R. DeBlasio, IEEE 1547 series of standards: interconnection issues, IEEE Transactions on Power Electronics 19 (5) (2004) 1159-1162. 
[175] S. Dhar, P. K. Dash, Harmonic profile injection-based hybrid active islanding detection technique for pv-vsc-based microgrid system, IEEE Transactions on Sustainable Energy 7 (4) (2016) 1473-1481. doi: 10.1109/TSTE. 2016.2515158

[176] S. Shrivastava, S. Jain, R. K. Nema, V. Chaurasia, Two level islanding detection method for distributed generators in distribution networks, International Journal of Electrical Power \& Energy Systems 87 (2017) 222-231.

[177] G. Bayrak, M. Cebeci, A communication based islanding detection method for photovoltaic distributed generation systems, International Journal of Photoenergy 2014.

[178] S. Syamsuddin, N. Rahim, J. Selvaraj, et al., Implementation of tms320f2812 in islanding detection for photovoltaic grid connected inverter, in: Technical Postgraduates (TECHPOS), 2009 International Conference for, IEEE, 2009, pp. 1-5.

[179] G. Bayrak, E. Kabalci, Implementation of a new remote islanding detection method for wind-solar hybrid power plants, Renewable and Sustainable Energy Reviews 58 (2016) 1-15.

[180] A. Hovanessian, M.-A. Norouzi, Islanding detection using wavelet transform and rate of change of frequency relay method in presence of different distributed generation technologies, IEEJ Transactions on Electrical and Electronic Engineering 11 (S1).

[181] A. Khamis, H. Shareef, E. Bizkevelci, T. Khatib, A review of islanding detection techniques for renewable distributed generation systems, Renewable and sustainable energy reviews 28 (2013) 483-493.

[182] J. Laghari, H. Mokhlis, M. Karimi, A. Bakar, H. Mohamad, Computational intelligence based techniques for islanding detection of distributed generation in distribution network: A review, Energy conversion and Management 88 (2014) 139-152.

[183] K.-H. Chao, M.-S. Yang, C.-P. Hung, Islanding detection method of a photovoltaic power generation system based on a CMAC neural network, Energies 6 (8) (2013) 4152-4169.

[184] I. Committee, et al., IEEE standard for interconnecting distributed resources with electric power systems, New York, NY: Institute of Electrical and Electronics Engineers.

[185] D. G. Photovoltaics, E. Storage, IEEE guide for design, operation, and integration of distributed resource island systems with electric power systems.

[186] Z. Yao, L. Xiao, Y. Yan, Seamless transfer of single-phase grid-interactive inverters between grid-connected and stand-alone modes, IEEE Transactions on Power Electronics 25 (6) (2010) 1597-1603.

[187] A. Micallef, M. Apap, C. Spiteri-Staines, J. M. Guerrero, Single-phase microgrid with seamless transition capabilities between modes of operation, IEEE Transactions on Smart Grid 6 (6) (2015) 2736-2745.

[188] S. S. Thale, V. Agarwal, Controller area network assisted grid synchronization of a microgrid with renewable energy sources and storage, IEEE Transactions on Smart Grid 7 (3) (2016) 1442-1452. 
[189] F. Tang, J. M. Guerrero, J. C. Vasquez, D. Wu, L. Meng, Distributed active synchronization strategy for microgrid seamless reconnection to the grid under unbalance and harmonic distortion, IEEE Transactions on Smart Grid 6 (6) (2015) 2757-2769.

[190] I. J. Balaguer, Q. Lei, S. Yang, U. Supatti, F. Z. Peng, Control for grid-connected and intentional islanding operations of distributed power generation, IEEE transactions on industrial electronics 58 (1) (2011) $147-157$.

[191] M. N. Arafat, A. Elrayyah, Y. Sozer, An effective smooth transition control strategy using droop-based synchronization for parallel inverters, IEEE Transactions on Industry Applications 51 (3) (2015) 24432454 .

[192] N. Lidula, A. Rajapakse, Voltage balancing and synchronization of microgrids with highly unbalanced loads, Renewable and Sustainable Energy Reviews 31 (2014) 907-920.

[193] S. A. Taher, M. Zolfaghari, C. Cho, M. Abedi, M. Shahidehpour, A new approach for soft synchronization of microgrid using robust control theory, IEEE Transactions on Power Delivery 32 (3) (2017) 1370-1381.

[194] S. Shah, H. Sun, D. Nikovski, J. Zhang, VSC-based active synchronizer for generators, IEEE Transactions on Energy Conversion 33 (1) (2018) 116-125.

[195] Abb, synchrocheck relay, product guide spau 140 c, 1mrs750421-mbg, ver: C/25.04.2006, 2006.

[196] Synchronism check relays, mlj, instructions manual, gek-106213c, ge power management. available at: ?http://www.gedigitalenergy.com/ products/man uals/mlj/mljman-c.pdf?

[197] Y. Sun, C. Zhong, X. Hou, J. Yang, H. Han, J. M. Guerrero, Distributed cooperative synchronization strategy for multi-bus microgrids, International Journal of Electrical Power \& Energy Systems 86 (2017) $18-28$.

[198] A. Bellini, S. Bifaretti, F. Giannini, A robust synchronization method for centralized microgrids, IEEE Transactions on Industry Applications 51 (2) (2015) 1602-1609.

[199] D. Shi, X. Chen, Z. Wang, X. Zhang, Z. Yu, X. Wang, D. Bian, A distributed cooperative control framework for synchronized reconnection of a multi-bus microgrid, IEEE Transactions on Smart Grid 9 (6) (2018) 6646-6655.

[200] D. Das, G. Gurrala, U. J. Shenoy, Linear quadratic regulator based bumpless transfer in microgrids, IEEE Transactions on Smart Grid 9 (1) (2018) 416-425.

[201] A. H. Etemadi, R. Iravani, Supplementary mechanisms for smooth transition between control modes in a microgrid, Electric Power Systems Research 142 (2017) 249-257.

[202] B. Das, V. Mukherjee, D. Das, DG placement in radial distribution network by symbiotic organisms search algorithm for real power loss minimization, Applied Soft Computing 49 (2016) 920-936.

[203] N. K. Roy, H. R. Pota, M. A. Mahmud, Dg integration issues in unbalanced multi-phase distribution networks, in: 2016 Australasian Universities Power Engineering Conference (AUPEC), 2016, pp. 1-5. doi:10.1109/AUPEC.2016.7749381. 
[204] T. Adefarati, R. C. Bansal, Integration of renewable distributed generators into the distribution system: a review, IET Renewable Power Generation 10 (7) (2016) 873-884. doi:10.1049/iet-rpg.2015.0378.

[205] R. C. Dugan, T. E. McDermott, G. J. Ball, Planning for distributed generation, IEEE industry applications magazine 7 (2) (2001) 80-88.

[206] H. L. Willis, Distributed power generation: planning and evaluation, Crc Press, 2000.

[207] L. Arya, A. Koshti, S. Choube, Distributed generation planning using differential evolution accounting voltage stability consideration, International journal of electrical power \& energy systems 42 (1) (2012) 196-207.

[208] T. Gözel, M. H. Hocaoglu, An analytical method for the sizing and siting of distributed generators in radial systems, Electric Power Systems Research 79 (6) (2009) 912-918.

[209] V. Murthy, A. Kumar, Comparison of optimal dg allocation methods in radial distribution systems based on sensitivity approaches, International Journal of Electrical Power \& Energy Systems 53 (2013) 450-467.

[210] D. Q. Hung, N. Mithulananthan, K. Y. Lee, Optimal placement of dispatchable and nondispatchable renewable DG units in distribution networks for minimizing energy loss, International Journal of Electrical Power \& Energy Systems 55 (2014) 179-186.

[211] D. Popović, J. Greatbanks, M. Begović, A. Pregelj, Placement of distributed generators and reclosers for distribution network security and reliability, International Journal of Electrical Power \& Energy Systems 27 (5-6) (2005) 398-408.

[212] A. Saif, V. R. Pandi, H. Zeineldin, S. Kennedy, Optimal allocation of distributed energy resources through simulation-based optimization, Electric Power Systems Research 104 (2013) 1-8.

[213] M. M. Elnashar, R. El Shatshat, M. M. Salama, Optimum siting and sizing of a large distributed generator in a mesh connected system, Electric Power Systems Research 80 (6) (2010) 690-697.

[214] M. P. HA, P. D. Huy, V. K. Ramachandaramurthy, A review of the optimal allocation of distributed generation: Objectives, constraints, methods, and algorithms, Renewable and Sustainable Energy Reviews 75 (2017) 293-312.

[215] A. Colmenar-Santos, C. Reino-Rio, D. Borge-Diez, E. Collado-Fernández, Distributed generation: A review of factors that can contribute most to achieve a scenario of dg units embedded in the new distribution networks, Renewable and Sustainable Energy Reviews 59 (2016) 1130-1148.

[216] M. Moradi, M. Abedini, A novel method for optimal DG units capacity and location in microgrids, International Journal of Electrical Power \& Energy Systems 75 (2016) 236-244.

[217] B. Poornazaryan, P. Karimyan, G. Gharehpetian, M. Abedi, Optimal allocation and sizing of DG units considering voltage stability, losses and load variations, International Journal of Electrical Power \& Energy Systems 79 (2016) 42-52. 
[218] S. Kansal, V. Kumar, B. Tyagi, Hybrid approach for optimal placement of multiple DGs of multiple types in distribution networks, International Journal of Electrical Power \& Energy Systems 75 (2016) 226-235.

[219] M. H. Moradi, S. R. Tousi, M. Abedini, Multi-objective pfde algorithm for solving the optimal siting and

[230] I. Kim, Optimal capacity of storage systems and photovoltaic systems able to control reactive power using the sensitivity analysis method, Energy 150 (2018) 642-652.

[231] S. Upadhyay, M. Sharma, A review on configurations, control and sizing methodologies of hybrid energy systems, Renewable and Sustainable Energy Reviews 38 (2014) 47-63. 
[237] J. Hu, J. Zhu, D. G. Dorrell, J. M. Guerrero, Virtual flux droop method: A new control strategy of inverters in microgrids, IEEE Transactions on Power Electronics 29 (9) (2014) 4704-4711.

[238] M. S. Golsorkhi, D. D. Lu, A control method for inverter-based islanded microgrids based on VI droop

[239] T. L. Vandoorn, B. Meersman, L. Degroote, B. Renders, L. Vandevelde, A control strategy for islanded microgrids with dc-link voltage control, IEEE Transactions on Power Delivery 26 (2) (2011) 703-713.

[240] W. Yao, M. Chen, J. Matas, J. M. Guerrero, Z.-M. Qian, Design and analysis of the droop control method for parallel inverters considering the impact of the complex impedance on the power sharing, IEEE Transactions on Industrial Electronics 58 (2) (2011) 576-588.

[241] J. He, Y. W. Li, Analysis, design, and implementation of virtual impedance for power electronics interfaced distributed generation, IEEE Transactions on Industry Applications 47 (6) (2011) 2525-2538.

[242] Q.-C. Zhong, Y. Zeng, Universal droop control of inverters with different types of output impedance, IEEE Access 4 (2016) 702-712.

[243] J. He, Y. W. Li, J. M. Guerrero, F. Blaabjerg, J. C. Vasquez, An islanding microgrid power sharing approach using enhanced virtual impedance control scheme, IEEE Transactions on Power Electronics 28 (11) (2013) 5272-5282.

[244] Y. Han, H. Li, P. Shen, E. A. A. Coelho, J. M. Guerrero, Review of active and reactive power sharing strategies in hierarchical controlled microgrids, IEEE Transactions on Power Electronics 32 (3) (2017) $2427-2451$.

[245] F. S. Gazijahani, J. Salehi, Robust design of microgrids with reconfigurable topology under severe uncertainty, IEEE Transactions on Sustainable Energy 9 (2) (2018) 559-569. 
[246] A. Khodaei, S. Bahramirad, M. Shahidehpour, Microgrid planning under uncertainty, IEEE Transactions on Power Systems 30 (5) (2015) 2417-2425.

[247] H. Wang, J. Huang, Cooperative planning of renewable generations for interconnected microgrids, IEEE Transactions on Smart Grid 7 (5) (2016) 2486-2496.

[248] A. Narayan, K. Ponnambalam, Risk-averse stochastic programming approach for microgrid planning under uncertainty, Renewable Energy 101 (2017) 399-408.

[249] L. Che, X. Zhang, M. Shahidehpour, A. Alabdulwahab, A. Abusorrah, Optimal interconnection planning of community microgrids with renewable energy sources, IEEE Transactions on Smart Grid 8 (3) (2017) 1054-1063.

[250] C. Stevanoni, Z. De Grève, F. Vallée, O. Deblecker, Long-term planning of connected industrial microgrids: a game theoretical approach including daily peer-to-microgrid exchanges, IEEE Transactions on Smart Grid (2018).

[251] S. F. Rafique, Z. Jianhua, Energy management system, generation and demand predictors: a review, IET Generation, Transmission \& Distribution 12 (3) (2017) 519-530.

[252] D. Lazos, A. B. Sproul, M. Kay, Optimisation of energy management in commercial buildings with weather forecasting inputs: A review, Renewable and Sustainable Energy Reviews 39 (2014) 587-603.

[253] U. K. Das, K. S. Tey, M. Seyedmahmoudian, S. Mekhilef, M. Y. I. Idris, W. Van Deventer, B. Horan, A. Stojcevski, Forecasting of photovoltaic power generation and model optimization: A review, Renewable and Sustainable Energy Reviews 81 (2018) 912-928.

[254] C. Bennett, R. A. Stewart, J. Lu, Autoregressive with exogenous variables and neural network short-term load forecast models for residential low voltage distribution networks, Energies 7 (5) (2014) 2938-2960.

[255] A. G. Abdullah, G. M. Suranegara, D. L. Hakim, Hybrid PSO-ANN application for improved accuracy of short term load forecasting, WSEAS Transactions on Power Systems 9 (446) (2014) 51.

[256] J. Dowell, P. Pinson, Very-short-term probabilistic wind power forecasts by sparse vector autoregression, IEEE Transactions on Smart Grid 7 (2) (2016) 763-770.

[257] V. Hoolohan, A. S. Tomlin, T. Cockerill, Improved near surface wind speed predictions using gaussian process regression combined with numerical weather predictions and observed meteorological data, Renewable Energy 126 (2018) 1043-1054.

[258] O. Abedinia, N. Amjady, N. Ghadimi, Solar energy forecasting based on hybrid neural network and improved metaheuristic algorithm, Computational Intelligence 34 (1) (2018) 241-260.

[259] L. Gigoni, A. Betti, E. Crisostomi, A. Franco, M. Tucci, F. Bizzarri, D. Mucci, Day-ahead hourly forecasting of power generation from photovoltaic plants, IEEE Transactions on Sustainable Energy 9 (2) (2018) 831-842. 
[260] M. Rafiei, T. Niknam, J. Aghaei, M. Shafie-khah, J. P. Catalão, Probabilistic load forecasting using an improved wavelet neural network trained by generalized extreme learning machine, IEEE Transactions on Smart Grid (2018).

[261] J. Lago, F. De Ridder, B. De Schutter, Forecasting spot electricity prices: Deep learning approaches and empirical comparison of traditional algorithms, Applied Energy 221 (2018) 386-405.

[262] N. Hatziargyriou, Microgrids: architectures and control, John Wiley \& Sons, 2014.

[263] M. A. Hossain, H. R. Pota, S. Squartini, A. F. Abdou, Modified PSO algorithm for real-time energy management in grid-connected microgrids, Renewable Energy 136 (2019) 746-757.

[264] D. Quiggin, S. Cornell, M. Tierney, R. Buswell, A simulation and optimisation study: Towards a decentralised microgrid, using real world fluctuation data, Energy 41 (1) (2012) 549-559.

[265] C. Corchero, M. Cruz-Zambrano, F.-J. Heredia, et al., Optimal energy management for a residential microgrid including a vehicle-to-grid system, IEEE transactions on smart grid 5 (4) (2014) 2163-2172.

[266] Y. Riffonneau, S. Bacha, F. Barruel, S. Ploix, Optimal power flow management for grid connected PV systems with batteries, IEEE Transactions on Sustainable Energy 2 (3) (2011) 309-320.

[267] M. Boaro, D. Fuselli, F. De Angelis, D. Liu, Q. Wei, F. Piazza, Adaptive dynamic programming algorithm for renewable energy scheduling and battery management, Cognitive Computation 5 (2) (2013) 264-277.

[268] G. Shi, Q. Wei, D. Liu, Optimization of electricity consumption in office buildings based on adaptive dynamic programming, Soft Computing (2016) 1-11.

[269] S. Squartini, D. Fuselli, M. Boaro, F. De Angelis, F. Piazza, Home energy resource scheduling algorithms and their dependency on the battery model, in: Computational Intelligence Applications In Smart Grid (CIASG), 2013 IEEE Symposium on, IEEE, 2013, pp. 122-129.

[270] G. K. Venayagamoorthy, R. K. Sharma, P. K. Gautam, A. Ahmadi, Dynamic energy management system for a smart microgrid, IEEE Transactions on Neural Networks and Learning Systems 27 (8) (2016) 16431656.

[271] A. Azizivahed, E. Naderi, H. Narimani, M. Fathi, M. R. Narimani, A new bi-objective approach to energy management in distribution networks with energy storage systems, IEEE Transactions on Sustainable Energy 9 (1) (2018) 56-64.

[272] W. Shi, N. Li, C.-C. Chu, R. Gadh, Real-time energy management in microgrids, IEEE Transactions on Smart Grid 8 (1) (2017) 228-238.

[273] D. S. Kirschen, G. Strbac, Fundamentals of power system economics, John Wiley \& Sons, 2004.

[274] H. B. da Silva, L. P. Santiago, On the trade-off between real-time pricing and the social acceptability costs of demand response, Renewable and Sustainable Energy Reviews 81 (2018) 1513-1521.

[275] P. Palensky, D. Dietrich, Demand side management: Demand response, intelligent energy systems, and smart loads, IEEE transactions on industrial informatics 7 (3) (2011) 381-388. 
[288] M. Alam, Enabling cooperative and negotiated energy exchange in remote communities, Ph.D. thesis, University of Southampton (2013).

[289] W. Tushar, C. Yuen, H. Mohsenian-Rad, T. Saha, H. V. Poor, K. L. Wood, Transforming energy networks via peer to peer energy trading: Potential of game theoretic approaches, arXiv preprint arXiv:1804.00962 (2018).

[290] B. Zhang, Q. Li, L. Wang, W. Feng, Robust optimization for energy transactions in multi-microgrids under uncertainty, Applied Energy 217 (2018) 346-360. 
[291] C. Yuen, A. Oudalov, The feasibility and profitability of ancillary services provision from multi-microgrids, in: Power Tech, 2007 IEEE Lausanne, IEEE, 2007, pp. 598-603.

[292] N. J. Gil, J. P. Lopes, Exploiting automated demand response generation and storage capabilities for hierarchical frequency control in islanded multi-microgrids, in: Proceedings of PSCC2008, 16th Power System Computation Conference, 2008.

[293] T.-T. Nguyen, H.-J. Yoo, H.-M. Kim, A droop frequency control for maintaining different frequency qualities in stand-alone multi-microgrid system, IEEE Transactions on Sustainable Energy 9 (2) (2018) 599-609.

[294] A. Madureira, J. Pereira, N. Gil, J. P. Lopes, G. Korres, N. Hatziargyriou, Advanced control and management functionalities for multi-microgrids, European Transactions on Electrical Power 21 (2) (2011) 1159-1177.

[295] H. Wang, J. Huang, Incentivizing energy trading for interconnected microgrids, IEEE Transactions on Smart Grid 9 (4) (2018) 2647-2657.

[296] W.-Y. Chiu, H. Sun, H. V. Poor, A multiobjective approach to multimicrogrid system design, IEEE Transactions on Smart Grid 6 (5) (2015) 2263-2272.

[297] S. A. Arefifar, M. Ordonez, Y. A.-R. I. Mohamed, Energy management in multi-microgrid systems?development and assessment, IEEE Transactions on Power Systems 32 (2) (2017) 910-922.

[298] Y. Liu, Y. Li, H. B. S. Gooi, J. Ye, H. Xin, X. Jiang, J. Pan, Distributed robust energy management of a multi-microgrid system in the real-time energy market, IEEE Transactions on Sustainable Energy 99 (2017) 1-1.

[299] M. Xie, X. Ji, X. Hu, P. Cheng, Y. Du, M. Liu, Autonomous optimized economic dispatch of active distribution system with multi-microgrids, Energy 153 (2018) 479-489.

[300] Y. Du, Z. Wang, G. Liu, X. Chen, H. Yuan, Y. Wei, F. Li, A cooperative game approach for coordinating multi-microgrid operation within distribution systems, Applied Energy 222 (2018) 383-395.

[301] F. H. Aghdam, J. Salehi, S. Ghaemi, Contingency based energy management of multi-microgrid based distribution network, Sustainable Cities and Society (2018).

[302] C. Zhang, J. Wu, Y. Zhou, M. Cheng, C. Long, Peer-to-peer energy trading in a microgrid, Applied Energy 220 (2018) 1-12.

[303] V. C. Gungor, D. Sahin, T. Kocak, S. Ergut, C. Buccella, C. Cecati, G. P. Hancke, Smart grid technologies: Communication technologies and standards, IEEE transactions on Industrial informatics 7 (4) (2011) $529-539$.

[304] Z. Guo, Z. J. Wang, A. Kashani, Home appliance load modeling from aggregated smart meter data, IEEE Transactions on power systems 30 (1) (2015) 254-262. 
[305] R. Pereira, J. Figueiredo, R. Melicio, V. M. F. Mendes, J. Martins, J. Quadrado, Consumer energy management system with integration of smart meters, Energy Reports 1 (2015) 22-29.

[306] Y. Sun, L. Lampe, V. W. Wong, Smart meter privacy: Exploiting the potential of household energy storage units, IEEE Internet of Things Journal 5 (1) (2018) 69-78.

[307] G. Giaconi, D. Gündüz, H. V. Poor, Smart meter privacy with renewable energy and an energy storage device, IEEE Transactions on Information Forensics and Security 13 (1) (2018) 129-142.

[308] M. Zanetti, E. Jamhour, M. Pellenz, M. Penna, V. Zambenedetti, I. Chueiri, A tunable fraud detection system for advanced metering infrastructure using short-lived patterns, IEEE Transactions on Smart Grid (2017).

[309] M. N. Kurt, Y. Yılmaz, X. Wang, Distributed quickest detection of cyber-attacks in smart grid, IEEE Transactions on Information Forensics and Security 13 (8) (2018) 2015-2030.

[310] L. Sankar, S. R. Rajagopalan, S. Mohajer, Smart meter privacy: A theoretical framework, IEEE Transactions on Smart Grid 4 (2) (2013) 837-846.

[311] C.-C. Sun, A. Hahn, C.-C. Liu, Cyber security of a power grid: State-of-the-art, International Journal of Electrical Power \& Energy Systems 99 (2018) 45-56.

[312] C. Marnay, H. Asano, S. Papathanassiou, G. Strbac, Policymaking for microgrids, IEEE Power and Energy Magazine 6 (3) (2008) $66-77$.

[313] M. Soshinskaya, W. H. Crijns-Graus, J. M. Guerrero, J. C. Vasquez, Microgrids: Experiences, barriers and success factors, Renewable and Sustainable Energy Reviews 40 (2014) 659-672.

[314] A. Ali, W. Li, R. Hussain, X. He, B. W. Williams, A. H. Memon, Overview of current microgrid policies, incentives and barriers in the european union, united states and china, Sustainability 9 (7) (2017) 1146. 